\title{
Teoría de la Cohesión Circular en el Matrimonio $y$ las Redes Sociales*
}

\author{
DOUGLAS R. WHITE \\ School of Social Sciences, Universidad de California, Irvine (CA) \\ drwhite@uci.edu
}

\section{INTRODUCCIÓN}

¿Cuáles son las formas básicas de reciprocidad por las que el matrimonio construye lazos fuertes y formas de integración social? Lévi-Strauss (1969[1949]) clasificó las formas de los ciclos matrimoniales elementales creados por el matrimonio entre primos en términos de sus implicaciones para la cohesión social, esto es, cómo los patrones matrimoniales integran grupos sociales. La reciprocidad puede tomar la forma de un ciclo de intercambio directo, bien dilatado, como en $\mathrm{A} \rightarrow \mathrm{B} \rightarrow \mathrm{A}$, bien inmediato, como en $\mathrm{A} \leftrightarrow \mathrm{B}$. Alternativamente, puede tomar la forma de un ciclo de intercambio indirecto o generalizado, $\mathrm{A} \rightarrow \mathrm{B} \rightarrow \mathrm{C} \ldots \rightarrow \mathrm{A}$. Esta clase de cuestión lleva a examinar los patrones actuales por los que los lazos matrimoniales entre familias crean algunos modelos observables de redes sociales, especialmente aquéllos que implican ciclos.

* Traducción de Beatriz Mañas. Reedición con la autorización de Mathématiques et Sciences Humaines / Mathematics and Social Sciences. Ref.: White, D. R.: «Ring cohesion Theory in marriage and social networks», Mathématiques et Sciences Humaines / Mathematics and Social Sciences, $n .^{\circ} 168,2004(4)$, pp. 59-82. A pesar de que el concepto de red se ha venido aplicando al análisis social desde finales de los años cincuenta, no es frecuente su aparición dentro de la bibliografía sociológica y antropológica en castellano. El propio autor de la teoría presentada en este artículo admite no tener conocimiento sobre alusiones a la misma en lengua castellana previas a la presente traducción. De ahí que se hayan planteado ciertas cuestiones a la hora de interpretar y traducir correctamente algunos de los términos del texto. Este ha sido el caso de la denominación de la propia teorra, Ring cohesion Theory, pues a pesar de que la traducción inmediata del término anglosajón ring es «anillo», se ha optado finalmente por la traducción "Teoría de la Cohesión circular», dada la dificultad de mantener dicha literalidad en castellano. Con esta traducción, que ha sido validada por el propio autor, hemos considerado que se mantiene de una forma sencilla el sentido de circularidad implícito en el término original en inglés, aunque ello no obste para tener en cuenta la capacidad metonímica de la palabra «anillo» en referencia al matrimonio, máxime cuando el objetivo de la teorfa es mostrar la contribución de los ciclos matrimoniales a la cohesión social. No obstante, cuando el término ring no aparece en el texto con una función de adjetivo, sino como un sustantivo, se mantiene la traducción más literal: «anillo». N.T. 
Señalando el inconveniente planteado por Goldenwiser [1913] acerca de la imposible complejidad de las redes de parentesco, Lévi-Strauss [1969, p. 125] arguía que, debido a que los seres humanos no pueden reconocer esta «aparente e imposible complejidad» en los patrones reticulares de los sistemas de parentesco, deben formular sus modelos de estructura social como el analista de las organizaciones sociales, en forma de reglas. Reglas y estrategias para usarlas, que podrían así formar un juego en el que las estructuras sociales sean vistas en evolución por las transformaciones de las reglas y cómo éstas son empleadas o aplicadas. La «estructura» define las reglas y constreñimientos de los juegos, y las estrategias se forman de acuerdo a ella [White, 1999]. Esto queda como un poderoso modelo de evolución y estructura social. Este estudio muestra cómo usar el análisis de redes para explicar las preferencias matrimoniales que no se basan necesariamente en reglas discretas, y cómo dar cuenta estadística de un modo más poderoso y útil de las preferencias que incorporan tanto reglas discretas como preferencias probabilísticas. Ello ofrece una explicación más precisa y conceptualmente intuitiva de la complejidad que subyace a las redes de parentesco y su formación, así como de las formas de cohesión que resultan de las dinámicas de parentesco.

Las estructuras elementales fueron para Lévi-Strauss las formuladas por reglas que tomaban una forma cerrada: una regla del matrimonio para una clase de parientes con los que uno se debe casar. Las estructuras «semicomplejas» eran aquellas que especificaban una clase de proscripción sobre aquellos con los que uno no se debe casar, lo suficientemente general y organizada como para implicar como efecto una clase de relaciones casaderas, también de formulación cerrada. Los «sistemas complejos» eran aquéllos que señalaban tendencias meramente estadísticas, preferencias o elusiones sobre aquéllos con los que casarse o no.

El estudio de los ciclos en una red reabre la cuestión sobre la complejidad de una manera muy diferente a la presuposición del análisis estructural, la cual sostiene que la complejidad descansa en la naturaleza de la regla, mecánica, estadística o intermedia. El análisis de redes aplica análisis estadístico a la red misma como una entidad compleja, independientemente de cómo las «reglas del juego» son aprehendidas por los antropólogos o articuladas por los actores. La consecuencia es una gran variedad de resultados sobre los tipos de complejidad. Este enfoque - desarrollado por Houseman y White [1998a y 1998b; White y Houseman, 2002] en una «théorie de la pratique» aplicada a las redes de parentesco- es también necesario en cualquier estudio de los efectos estadísticos de las diferentes variables sobre las elecciones matrimoniales.

Un modo de estudiar la contribución de los ciclos matrimoniales a la integración social y a las formas de reciprocidad - y cómo las relaciones anteriores afectan a los actuales matrimonios-consiste en desarrollar un cálculo de la ocurrencia de tales ciclos en una red matrimonial. Los intentos tempranos de hacer esto, como el análisis de los ciclos matrimoniales de los Purum realizado por Das (1945), fracasaron en el intento de desarrollar un cálculo adecuado y fueron 
criticados por Schneider (1965) ${ }^{1}$. Uno de los objetivos del presente artículo, inserto en una teoría de la cohesión circular, es desarrollar un cálculo apropiado para las redes de parentesco y de matrimonio.

\subsection{Definiciones y Teoremas}

Para proponer cuestiones básicas de investigación se requieren definiciones teóricas sobre grafos. Un digrafo (digraph) $\mathrm{D}=\langle\mathrm{V}, \mathrm{P}, \mathrm{T}\rangle$ es un conjunto $\mathrm{V}$ de nodos (vértices) y un conjunto $\mathrm{P}$ de pares ordenados en $\mathrm{V}$ clasificados por un conjunto $\mathrm{T}$ de tipos de pares. Los diferentes tipos $k=1, t$ de pares de relaciones $\mathrm{P}$ en $(\mathrm{V} \times \mathrm{V})$ se denominan aristas (edges) si todos los pares de este tipo son lazos no dirigidos (p. ej., los hermanos) y arcos (arcs) si alguno de ellos es dirigido (p. ej., padre/hijo) ${ }^{2}$. Un grafo $\mathrm{G}=\langle\mathrm{V}, \mathrm{P}, \mathrm{T}\rangle$ es un digrafo con la restricción de que todas las relaciones $\mathrm{P}$ en $(\mathrm{V} \times \mathrm{V})$ son aristas. Un subgrafo de un grafo $\mathrm{G}$ es un grafo que tiene todos sus nodos y aristas en $\mathrm{G}$. Un subgrafo inducido $\langle\mathrm{S}\rangle$ de un grafo $\mathrm{G}$ es un subgrafo de $\mathrm{G}$ que tiene el conjunto de nodos $\mathrm{S}$ en $\mathrm{G}$ más todas las aristas de $\mathrm{G}$ en el subconjunto de pares $\langle\mathrm{S} \times \mathrm{S}\rangle$. Un camino (path) y un ciclo (cycle) son dos tipos de series de nodos y aristas alternantes en los que cada arista conecta los dos nodos a los que es adyacente en la serie: en un camino los nodos no se repiten; en un ciclo, el primer y último nodos son idénticos pero ningún otro nodo se repite. Un semiciclo (semicycle) de un digrafo es un ciclo en el que los arcos son tratados como aristas, conectando nodos en cualquier dirección. Un ciclo/camino dirigido (directed cycle/path) de un digrafo es un ciclo/camino cuyo orden de conexión de nodos es consistente con una dirección uniforme de los arcos. Un ciclo en un grafo inducido o digrafo -en el que las aristas son sustituidas por arcos- es equivalente a un semiciclo. Un grafo $G$ está conectado si todo par de nodos está unido por un camino. Un componente de $\mathrm{G}$ es cualquiera de sus mayores subgrafos conectados. El grado (degree) de un nodo en un grafo $\mathrm{G}$ es el número de aristas incidentes hacia el nodo. Un ciclo simple (simple cycle) en un grafo es un subgrafo inducido $<\mathrm{S}>$ que contiene un único ciclo; y todos los nodos en $\mathrm{S}$ tienen grado dos. Un árbol (tree) es un grafo sin ciclos.

Si representamos los datos de una red dada de parentesco y matrimonio dentro de este marco, un p-grafo (p-graph) es un digrafo en el cual los matri-

${ }^{1}$ Los antropólogos anglo-americanos de orientación empírica tendían a asustarse de tales estudios siguiendo la crítica de Schneider o criticando los supuestos estructuralistas, mientras los antropólogos franceses continuaron estudiando las redes matrimoniales y de parentesco usando el vocabulario de un marco lógico-deductivo de pensamiento estructural y sin adoptar un enfoque reticular para el análisis empírico.

${ }^{2}$ Los términos de los que se ofrece una definición formal están en cursiva, siguiendo generalmente los de Haray (1969), aunque estos digrafos, asumiendo que un par de nodos sólo tiene un tipo de arista o arco (p. ej., uno no debe casarse con su padre), añaden una clasificación de tipos de arcos y aristas. 
monios son considerados nodos, y los arcos son aquéllos que se encuentran entre los padres en una generación ascendente y sus hijos o los matrimonios de los hijos en una generación descendente. Los arcos se distinguen por el sexo del hijo/a ${ }^{3}$. Se consideran los inventarios de tipos de matrimonio que tienen lugar en un p-grafo definiendo cada uno de los tipos como un tipo distintivo o anillo (ring), o simple semiciclo en el p-grafo $\langle\mathrm{V}, \mathrm{P}, \mathrm{T}\rangle$. Cada tipo de anillo identifica un matrimonio empírico de ese tipo como un subgrafo inducido, o tipo de fragmento (fragment) dentro de la red empírica. Un anillo puede estar compuesto de varias clases diferentes de elementos en $\langle\mathrm{V}, \mathrm{P}, \mathrm{T}\rangle$ que forman un semiciclo. Un fragmento correspondiente de $\mathrm{G}$ debe consistir en un subgrafo inducido que es isomorfo al anillo. El anillo nulo (null ring) es aquél en el cual el cierre cíclico del anillo es un enlace nulo, indicando un matrimonio en el que no hay un enlace de un p-grafo anterior entre esposos. Excepto para el anillo nulo, un matrimonio empírico o un tipo de anillo que crea un semiciclo se denomina reencadenamiento** o matrimonio reencadenado ${ }^{4}$.

Un único matrimonio empírico puede ser de uno o más tipos de anillos, y muchos tipos diferentes de anillos -aunque no todos- pueden ocurrir conjuntamente. Se muestra un ejemplo en la Figura 1, un diagrama genealógico con triángulos para los hombres, círculos para las mujeres y cuadrados para los progenitores no especificados. Para convertir esto en un p-grafo, se dibuja cada pareja junta dentro de un único nodo, como en la Figura 2. El matrimonio con la etiqueta $A$ en el diagrama (a) de ambas figuras es de dos tipos diferentes: intercambio de hermanas con $\mathrm{B}$ y FaBrDa. Esta combinación de dos matrimonios reencadenados, uno consanguíneo y otro no, lleva consigo un segundo matrimonio consanguíneo para $\mathrm{B}$, también del tipo FaBrDa. Alternativamente, los dos matrimonios $\mathrm{FaBrDa}$ (A, B) conllevan intercambio de hermanas. Se muestra otro ejemplo en el p-grafo (b): el matrimonio $A$ es un intercambio de hermanas con D. La consecuencia es que los matrimonios $\mathrm{C}$ y $\mathrm{D}$ son también de ambos tipos MoBrDa y FaSiDa. En el p-grafo (c), el matrimonio A es un intercambio de her-

${ }^{3}$ La convención de considerar matrimonios como nodos en los p-grafos fue adoptada por Weil (1949) para la representación algebraica de modelos de parentesco, generalizada por Bertin (1983) a las genealogías, continuada por Jorion (1984) para clases de modelos de parentesco más amplias, y vuelta a generalizar por White y Jorion $(1992,1996)$ a las genealogías. Los p-grafos no distinguen medio-hermanos porque cuando el mismo progenitor está en dos matrimonios diferentes, la relación de los medio-hermanos se parece a la de los primos. Los medio-hermanos se distinguen en el formato bipartito del p-grafo, en el cual los individuos son un conjunto de nodos y las parejas otro, y en los grafos más convencionales con individuos como nodos y con arcos desde los padres hasta los hijos. Los últimos incluyen el formato de cálculo matrimonial descrito aquí y por Hamberger et al. (2004), y el grafo Ore (1960) más primitivo que carece de encadenamientos para los matrimonios.

** El término relinking aparecerá traducido en lo sucesivo como «reencadenamiento/s», de forma similar a la solución adoptada en lengua francesa («renchaînement») [Ver White, D. R. «Ring cohesion Theory in marriage and social networks". Mathématiques et Sciences Humaines / Mathematics and Social Sciences, n. ${ }^{\circ}$ 168, 2004(4), p. 59)]. Ello se debe a que dicha expresión ha sido utilizada desde la antropología para describir los encadenamientos sucesivos de matrimonios que conforman una red de parentesco. N.T.

4 Jola, Verdier y Zonabend (1970). 


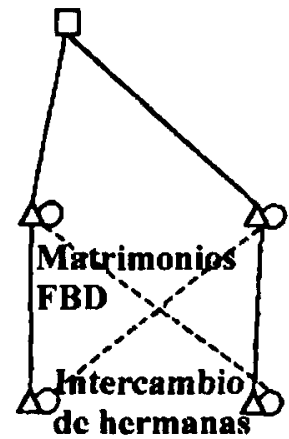

A

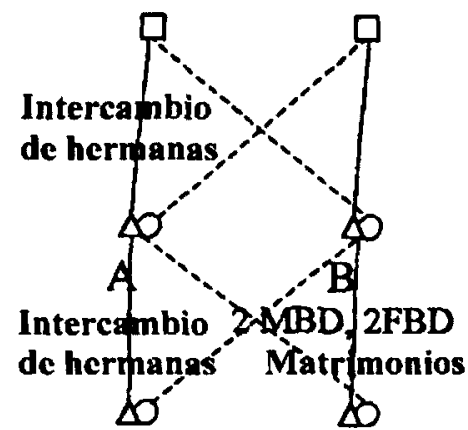

C

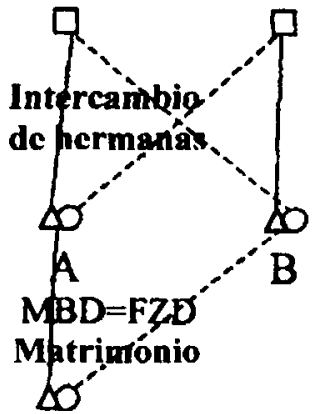

$\mathrm{C}$ (a) (b) (c)

Figura 1. Tipos de matrimonios reencadenados con dos o tres ciclos independientes.

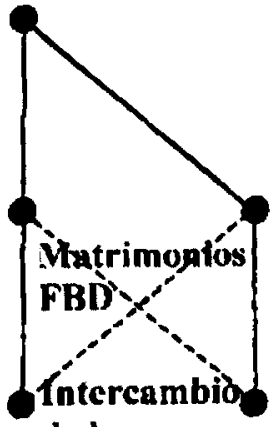

de hermanas
B

(a)

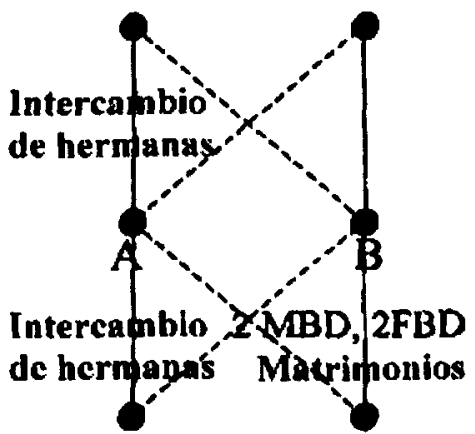

$\mathrm{C}$
D

(b)

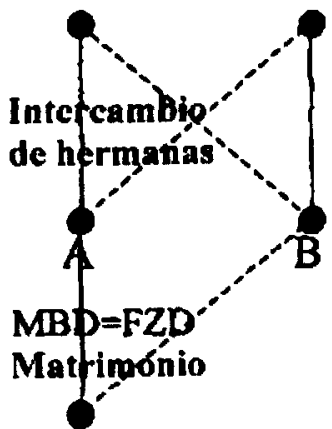

C

Figura 2. Los tipos de matrimonios reencadenados de la figura 1 dibujados como p-grafos.

manas para B, y un segundo matrimonio $\mathrm{C}$ es de los tipos MoBrDa y FaSiDa, cada uno implicando al otro, dado el anterior intercambio de hermanas.

En la Figura 2, todas las parejas son reemplazadas por nodos únicos para los matrimonios, y ambos tipos de líneas - sólidas para los hombres y discontinuas para las mujeres- diferencian las relaciones genealógicas. La belleza y simplicidad de este (di)grafo, cuyas líneas son arcos dirigidos en el tiempo, consiste en que todos los tipos de matrimonios pueden ahora ser identificados estrictamente en términos de anillos o tipos de ciclos de matrimonios (semiciclos en un digrafo). El intercambio de hermanas, por ejemplo, es un grafo en forma de «pa- 
jarita» (bow-tie graph) para las generaciones antecedentes y precedentes con encadenamientos de un sexo en la vertical y del otro sexo en las diagonales.

Ahora podemos definir diferentes tipos de fragmentos de grafos, tales como el modelo cíclico de intercambio de hermanas con forma de "pajarita", y estos fragmentos de anillo pueden buscarse y enumerarse en una base de datos de redes. La forma en la que esto puede hacerse eficientemente con un software de redes se discutirá más adelante. Esto produce una frecuencia de distribución para la incidencia de distintos tipos de matrimonio. En los ejemplos de las Figuras (a), (b) y (c), hay tres, seis y tres tipos diferentes de matrimonio, respectivamente ${ }^{5}$.

Las definiciones teóricas de grafos simplifican, de esta forma, ciertos problemas de análisis antes de plantear cuestiones de investigación. En estos ejemplos, es evidente que los ciclos en un grafo no son independientes. La Figura (a), por ejemplo, tiene tres tipos o ciclos de matrimonios (semiciclos digráficos): dos matrimonios $\mathrm{FaBrDa}$ y un intercambio de hermanas (matrimonio SiHuSi). La presencia de cualquiera de estos dos anillos trae consigo el tercero.

En teoría de grafos, un conjunto de ciclos independientes es cualquier conjunto de ciclos en el cual cada ciclo contiene al menos una arista que no está presente en ninguno de los otros ciclos. Debido a que un p-grafo contiene ciclos no dirigidos, el término ciclo usado en este y similares contextos se referirá a sus semiciclos, dejando la dimensión temporal implícita (pero identificada con las etiquetas apropiadas). En el ejemplo (a), dos ciclos cualesquiera son independientes, pero los tres tomados conjuntamente no son independientes porque todas las aristas en el grafo son usadas por cualquier par de ciclos.

TEOREMA i. Ciclos independientes (cohesión circular). El mayor número posible de ciclos independientes para un grafo $\mathrm{G}$ con $k$ aristas, $n$ nodos, y $d$ componentes desconectados es $k-n+d$. Esto se aplica a cualquier grafo $\mathrm{G}$ (ver la demostración [Haray, 1969]) ${ }^{6}$. En una red de parentesco representada por un p-grafo $\mathrm{G}$ conectado, hay un máximo de $r=k-n+1$ ciclos independientes. Para un grafo genealógico comparable donde los individuos son nodos y los arcos recorren desde los padres hasta los hijos (grafo Ore) ${ }^{7}$, la fórmula para el máximo número de ciclos matrimoniales independientes es $r=k-n+1-\Sigma_{k=1, s}\left(n_{s}-1\right)$ donde el subíndice $s$ es el número de grupos de hermanos con dos progenitores y $n_{s}$ es el tamaño de cada uno.

${ }^{5}$ Los intercambios de hermanas, como muchos reencadenamientos simétricos de dos familias, podrían también contarse como dos matrimonios del mismo tipo. Los cálculos usados aquí eliminan esas simetrías.

${ }^{6}$ La demostración es simple, en los $n-1$ nodos está el mínimo requerido para conectar un grafo, las aristas adicionales crearán, por definición, $k-(n-1)$ ciclos independientes, y cualquier número arbitrario de ciclos mayor que $k-n+1$ será no-independiente. Comenzando por los $d$ componentes (desconectados) de un grafo, se requerirán $n$ - $d$ aristas para conectar los nodos en cada componente, de forma que la fórmula será derivada de $k-(n-d)$ ciclos independientes.

7 Un gráfico Ore primitivo (1960) puede también enriquecerse distinguiendo los nodos individuales por sexo. 
Si se comprueba estas formulas en la Figura (a), $r=2$, mientras que para (b), $r=3$, y para (c), $r=2$ : hay un máximo de dos matrimonios independientes en los ejemplos (a) y (c) y de tres en (b). Recíprocamente, aunque no sea evidente de forma inmediata, el número mínimo de ciclos matrimoniales no independientes es uno en (a) y tres en (b) ${ }^{8}$.

Dada la forma en que se calculan los reencadenamientos, debido a que las frecuencias de fragmentos son calculadas por subgrafos inducidos que son ciclos simples, los ciclos no-independientes en un subgrafo inducido con $r=2$ no pueden resultar de la conjunción de dos matrimonios consanguíneos, o de dos matrimonios no consanguíneos, sino sólo de la combinación de uno de cada tipo, como en los ejemplos (a) y (c). Un matrimonio dado, por ejemplo, no puede ser a la vez un FaBrDa y un MoBrDa, a menos que (1) los padres de uno de ellos sean hermanos, o (2) una persona tenga más de un padre y/o madre. Bajo estas restricciones, un hombre puede casarse tanto con una FaBrDa como con una MoBrDa solamente si son esposas diferentes.

La teoría de la cohesión circular usa el cálculo de ciclos independientes para resolver los problemas de cómo explicar una estructura matrimonial compleja en términos de preferencias, cuya extensión máxima hasta determinado número de ciclos de matrimonio independientes es de tamaño $r$. Supone un modelo para una red con $r$ ciclos independientes, en el que se postula que tendrán lugar $k=r$ matrimonios debido a una regla de matrimonio preferencial, y donde los matrimonios preferenciales crean $k$ ciclos. Si se identifican $k=r$ matrimonios preferenciales, su presencia en la red cuenta para todos los demás ciclos, aunque las formas en que concatenan dentro de ciclos no-independientes está abierta a un estudio posterior sobre estructuras de segundo orden ${ }^{9}$. Los ciclos no-independientes se encuentran necesariamente concatenados desde algún conjunto de ciclos matrimoniales cuya extensión máxima es $r$.

En la teoría de grafos, para dar cuenta de otros ciclos se toma la unión de los subgrafos para dos ciclos, y se sustraen entonces las aristas que tienen en común. Esto generará un ciclo nuevo no-independiente. En la Figura (a), por ejemplo, la adición gráfica de los dos matrimonios $\mathrm{FaBrDa}$ deja como resultado el intercambio de hermanas. De forma similar, la adición gráfica de uno de los matrimonios $\mathrm{FaBrDa}$ con el intercambio de hermanas deja el otro matrimonio $\mathrm{FaBr}$ $\mathrm{Da}, \mathrm{y}$ así sucesivamente. El uso de estos procedimientos nos permite dar un cómputo de ciclos independientes en un grafo, con tal de que el modelo identifique esos tipos de matrimonios que, o bien son preferenciales, o bien no han sido permitidos debido una proscripción matrimonial.

${ }^{8}$ El cálculo del número total de ciclos en un grafo es complicado, pero puede determinarse a partir de la forma en que los pares de ciclos se superponen en un conjunto máximo de ciclos. Si dos ciclos independientes comparten una arista, crean un ciclo dependiente. Si comparten dos nodos pero ninguna arista, generan cuatro ciclos dependientes, y asr sucesivamente. No hay una fórmula fácil.

${ }^{9}$ En un bicomponente de un p-grafo con $m$ matrimonios, cada matrimonio con dos nodos parentales es un reencadenamiento y $m \geq r$, pero como muestra (b) en la Figura $3, m$ no siempre se iguala a $r, y$ el número total de ciclos puede ser mucho mayor que $m$.

EMPIRIA. Revista de Metodología de Ciencias Sociales. N. ${ }^{\circ}$ 10, julio-diciembre, 2005, pp. 37-69. ISSN: $1139-5737$ 
Supongamos ahora que un modelo en el que un cierto número $m$ de tipos de matrimonio pueda ser clasificado por orden de preferencias. Sea $f_{i}$ la frecuencia empírica del primero y cualquier otro tipo de matrimonio sucesivo $(i=1, m)$. Si extraemos cada uno de estos matrimonios de la red, observaremos típicamente una reducción de la frecuencia de otros tipos de matrimonio para casos en los que los matrimonios de este tipo se superponen con matrimonios de otros tipos. Sea $\mathrm{F}=\Sigma_{i=1, m} f_{i}$ el número total de matrimonios extraídos por este proceso. $\mathrm{Si}$ $\mathrm{F}=r$, no quedarán ciclos. Las eliminaciones reducen así el grafo genealógico hasta aproximarlo a un árbol (sin ciclos) ${ }^{10}$. Este modelo habrá tenido éxito en dar cuenta de todos los ciclos de la red. Para explicar los ciclos independientes y, más allá, de cómo se concatenan, se ofrece igualmente una explicación de los ciclos no-independientes.

\section{CUESTIONES A INVESTIGAR Y MÁS DEFINICIONES}

\section{Cuestiones a investigar}

Las cuestiones de interés que derivan de la teoría de la cohesión circular se aplican a cualquier red genealógica en la cual los individuos tienen en su mayoría dos padres (independientemente de cómo sean definidos y, por tanto, no necesariamente biológicos), y en la que las relaciones parentales y ancestrales están temporalmente ordenadas (padres que preceden a los hijos, de forma que ningún ciclo dirigido ocurre donde uno es el propio antepasado de uno mismo):

1. ¿Cuáles son las fuentes de la cohesión marital en la comunidad?

2. ¿Pude usarse el cálculo de la cohesión circular para ayudar a explicar las fuentes de cohesión en una red de parentesco debida a matrimonios reencadenados?

3. ¿Pueden enumerarse exactamente las frecuencias de los tipos matrimoniales hasta el límite del conocimiento del investigador tal como está codificado en la base de datos?

4. ¿Existe una forma válida para identificar qué conjuntos de matrimonios en cualquier comunidad dada son más preferenciales que el resto? ¿Cuál es la nomenclatura estadística de los conjuntos de matrimonios preferenciales?

5. ¿Qué quedará de una red empírica dada cuando esos matrimonios postulados como preferenciales sean sustraídos, y cómo se lleva esto a cabo?

${ }^{10}$ La demostración es obvia: si eliminamos $r=k-n+d$ aristas de un grafo con $k$ aristas, quedarán $k-r=n+d$ aristas; si ambos grafos, inicial y final, están conectados, entonces el grafo final será un árbol si $d=1$ y un conjunto de $d$ árboles es de otra forma. 
Las respuestas a estas cuestiones requieren más definiciones y teoremas construidos desde la base lógica de la teoría de la cohesión circular. Las notas a pie de página de las secciones siguientes envían estas preguntas y las refieren a operaciones del programa de análisis de redes Pajek [Batagekj, Mrvar 1998, 2002] usado con finalidad de cálculo.

\subsection{Cohesión global y fuentes de cohesión}

Volviendo al tema inicial, la cohesión social, los ciclos creados por el matrimonio en redes genealógicas crean las fronteras de la endogamia estructural [White, 1997], donde cada matrimonio conecta con cualquier otro a través de dos o más caminos independientes. Una representación a través de un p-grafo de las redes matrimoniales permite operacionalizar esta definición dando lugar a la cohesión social creada por el matrimonio. Dos o más caminos de un nodo a otro son definidos como (nodo-) independientes si no tienen nodos intermedios en común. El nivel de cohesión del grafo inducido de un p-grafo, medido por un número entero $k$, es definido como el número $\mathrm{k}$ de nodos que deben ser extraídos para desconectarlo. Este es el número ciclomático (cyclomatic number) o conectividad $k$ de un grafo.

TEOREMA ii. Multiconectividad (cohesión circular). Si un grafo o subgrafo tiene conectividad $\mathrm{k}$, entonces cada par de sus nodos tiene $\mathrm{k}$ caminos nodo-independientes entre ellos [Menger, 1927; Haray, White 2001], y viceversa.

Un p-grafo es regular si ningún individuo tiene más de dos padres y ningún nodo marital tiene más de dos parejas parentales, una para un hombre y otra para una mujer; bien sea casados o en pareja, el hombre y la mujer son miembros del mismo nodo en el p-grafo. Un bicomponente (bicomponent) de un p-grafo $\mathrm{P}$ es un subgrafo máximo de $\mathrm{P}$ (el mayor posible) en el que cada par de nodos está unido por dos o más caminos nodo-independientes y está contenido en un (semi) ciclo ${ }^{11}$. Ningún p-grafo pude tener conectividad 3 o superior si es regular. Un bi-

$"$ El cálculo de bicomponentes a través de Pajek se hace con Net/Component/Bicomponents. Los resultados son enviados a la ventana de Jerarquía que debe ser pulsada en el número ráz de la pantalla para ver la jerarquía de posibles bicomponentes superpuestos. Dos bicomponentes pueden compartir como mucho un nodo en común, y ello no está permitido a los ciclos de bicomponentes superpuestos porque, en su lugar, deben constituir bicomponentes sencillos. El comando Net/Component/Bicomponents también genera múltiples particiones en la ventana de particiones. La primera identifica vértices que pertenecen exactamente a un bicomponente por su número, con nodos que no están en ninguno de los bicomponentes asignados a la partición cero, y con nodos de intersección (puntos de articulación) entre bicomponentes asignados a la partición 99998. La segunda partición identifica puntos de articulación, asignando a cada uno el número de bicomponentes de los que son miembros. Para seleccionar el subgrafo consistente en nodos en cualquiera de los bicomponentes, utilizamos Operations/Extract from network/Partition, y dando entonces un valor mínimo de 1 y un valor máximo lo suficientemente grande, se obtendrá como resultado el subgrafo mostrado en la ventana reticular. 
componente de un p-grafo regular es, por tanto, una unidad máxima de endogamia estructural e isomorfa a la máxima unidad de cohesión social para una red genealógica.

De esta forma los p-grafos regulares proporcionan una forma natural de reconocer los grupos estructuralmente endógamos y genealógicamente cohesivos dentro de una red genealógica de parentesco y matrimonio. Una comunidad sexualmente reproductiva tendrá típicamente un único gran bicomponente o unidad estructuralmente endogámica. Alternativamente, una comunidad sexualmente reproductiva puede estar definida por los límites locales de la endogamia estructural, es decir, descontando los matrimonios de aquéllos que han emigrado permanentemente desde el área local y cuyos descendientes no han regresado.

$\mathrm{El}$ análisis de estructuras cohesivas se hace típicamente sobre un único bicomponente de tamaño máximo o sobre una unidad estructuralmente endógama de un p-grafo regular, al que nos referimos como subgrafo de «comunidad» cohesiva en una red genealógica. Solamente dentro de tal comunidad, o bicomponente del grafo de parentesco, podemos encontrar anillos.

\subsection{Cálculo de la Cohesión Circular}

Los matrimonios entre personas que están previamente relacionadas vienen en dos formas básicas, con subvariedades. Una es la de los matrimonios consanguíneos. La otra es el matrimonio entre parientes políticos, extendido genéricamente a todos aquéllos que están vinculados, antes de su matrimonio, por uno o más caminos que combinan relaciones de sangre y anteriores matrimonios. Ambos se llaman matrimonios reencadenados (relinking marriages). Cualquier tipo de matrimonio reencadenado, cuando se define por un ciclo simple, implicará 1, 2, 3 o un número mayor de matrimonios, incluyendo el matrimonio reencadenado que se encuentre en el último lugar de la secuencia temporal. El mínimo número de matrimonios implicados (descontando aquéllos de antepasados a otros nodos del anillo) es idéntico al número de familias que están reencadenadas, donde las familias se definen como el número de componentes (desconectados) que permanecen después de que los matrimonios que dan lugar a ciclos sean suprimidos.

Para emplear el método de extracción de matrimonios de la cohesión circular tal como se aplica a las redes genealógicas, la representación teórica del grafo debe incluir el matrimonio como un tipo de vínculo y, por tanto, a los individuos como nodos del grafo. Ya no basta con los P-grafos. En el tipo de grafo necesario, cuando todos los encadenamientos matrimoniales insertos en un tipo dado de ciclos matrimoniales o anillo son extraídos del grafo, los individuos conectados por el matrimonio deben permanecer ${ }^{12}$. Los formatos de las redes ge-

${ }^{12}$ En Pajek, el comando Nets/Firt network se usa para seleccionar la red principal y Nets/Second network para seleccionar la red con aristas o arcos que serán sustraídos. Nets/difference sustraerá los arcos y las aristas de la segunda red desde la primera, dejando todos los nodos.

EMPIRIA. Revista de Metodología de Ciencias Sociales. N. 10, julio-diciembre, 2005, pp. 37-69. 
nealógicas que conforman esta estructura también hacen posible la definición siguiente, según la cual una relación matrimonial (matrimonio, unión, pareja) puede existir sin hijos, pero cuando los hijos de una pareja existen se considera que «están casados» por propósitos analíticos.

Anillos matrimoniales ${ }^{13}$. Un anillo matrimonial en un digrafo genealógico con aristas para los vínculos matrimoniales entre los individuos y arcos desde los padres hasta los hijos, es un (semi)ciclo simple tal que, tras la extracción de las aristas todos los componentes son árboles arraigados con un único nodo ancestral. Un árbol es mínimo si consiste en un único nodo.

Los anillos de un digrafo genealógico junto a sus árboles y ramas permiten afirmar que un anillo matrimonial corresponde a un ciclo de matrimonios dentro o entre las familias, definidas por miembros de un árbol arraigado de parientes consanguíneos con un ancestro o pareja ancestral común. Destaquemos que «dentro o entre familias» es relativo a un anillo particular y no a un conjunto de anillos. En la Figura 2(a) había tres anillos dentro de una única familia: dos matrimonios FaBrDa y un intercambio de hermanas. El intercambio de hermanas es un ciclo simple porque no hay vínculos adicionales entre padre-hijo o marido-esposa entre los actores del ciclo, y este anillo, tomado en sí mismo, es un matrimonio reencadenado entre dos familias nucleares.

En otro ejemplo, en la Figura 3(a), sólo hay dos anillos para los matrimonios FaSiDa. El matrimonio FaBrSoWiSi no es un anillo debido al vínculo «línea 1» entre la mujer del centro y sus padres. Este vínculo impide que el matrimonio FaBrSoWiSi constituya un reencadenamiento entre dos familias. Si no hubiera existido, como en 3(b), el matrimonio FaBrSoWiSi sería un anillo, que encadenaría dos familias. El algoritmo que encuentra fragmentos como ciclos simples reconoce esta diferencia. El subgrafo inducido por los nodos del ciclo sobre el perímetro externo de la Figura 3(a) fracasa al constituir un anillo porque contiene, adicionalmente, el vínculo «línea 1 ». Recordemos que un subgrafo inducido $<\mathrm{S}\rangle$ de un digrafo es un subconjunto $\mathrm{S}$ de sus nodos más todas las aristas $\mathrm{y}$ arcos que están en el subconjuto de pares $\langle\mathrm{S} \times \mathrm{S}\rangle$. En un subgrafo de una red mayor inducida por su isomorfismo hacia un anillo, el grado de cada nodo debe ser dos porque el anillo es un ciclo simple. La Figura 3(b) se califica como anillo por este criterio, pero no así la Figura 3(a), que en su lugar contiene dos de tales anillos.

TEOREMa iii. Ramificación (cohesión circular ${ }^{14}$ ). Como puede comprobarse en general, y como se muestra en estos ejemplos, no hay más de dos ramas en nin-

${ }^{13}$ Esta sección y la definición final de los anillos matrimoniales es un trabajo conjunto con Klaus Hamberger y otros con la colaboración continuada del grupo de investigación TIME en París.

${ }_{14}$ Dado que el anillo matrimonial es un conjunto de uno o más árboles familiares arraigados con dos ramas, encadenados y reencadenados por lazos matrimoniales para forma un semiciclo simple, debe haber exactamente dos nodos dentro de cada árbol conectados por matrimonio. Si tiene lugar un anillo matrimonial entre familias, cada familia debe tener dos ramas para alcanzar los dos nodos que la conectan en el círculo de árboles familiares.

EMPIRIA. Revista de Metodología de Ciencias Sociales. N. ${ }^{\circ}$ 10, julio-diciembre, 2005, pp. 37-69. 


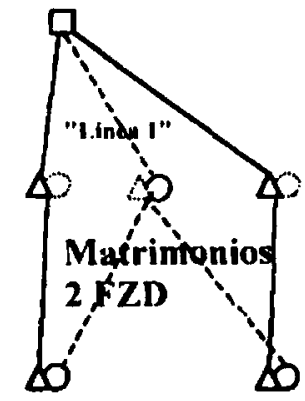

A
B

(a)

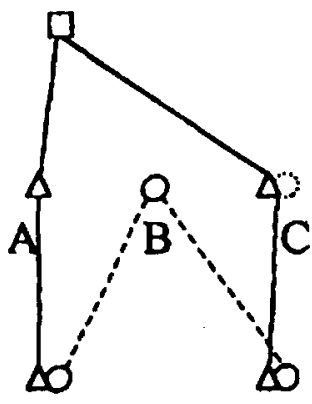

D

(b)

Figura 3. Redes genealógicas con (a) dos anillos y (b) un anillo.

guno de los árboles arraigados de un anillo matrimonial. En la red de la Figura 3(a) hay cuatro ramas y dos matrimonios reencadenando dos anillos dentro de una única familia. Sin embargo, cada anillo individual tiene únicamente dos ramas. Cada anillo FaSiDa en 3(a) tiene dos ramas; y cada uno de los anillos de FBD y de intercambio de hermanas en 1(a) tiene dos ramas hacia las subfamilias reencadenadas. El Teorema iii puede ser reformulado de una forma más intuitiva:

Un anillo matrimonial corresponde a un ciclo de matrimonios dentro o entre familias definidas como miembros de un árbol arraigado (rooted tree) de parientes consanguíneos que tienen un ancestro común y como mucho dos ramas.

Un anillo matrimonial mínimo puede consistir incluso en un ciclo de matrimonios con árboles arraigados mínimos consistentes en nodos únicos sin ramas. Cuatro individuos, por ejemplo, pueden formar un anillo matrimonial si un hombre se casa con su WiHuWi. Como ejemplo adicional, si se diera el caso de que los individuos A y B de la Figura 3(b) fueran los padres de D y E, entonces 3(b) no sería nunca más un anillo matrimonial porque las parejas A-C-E formarían un anillo matrimonial $\mathrm{FaBrDa}$ entre ellas mismas, del cual $\mathrm{D}$ no forma parte.

\section{ENUMERACIÓN}

Muchos etnógrafos han señalado la importancia de la relación de hermanos totales frente a la de medio-hermanos en las sociedades humanas. Dado que las parejas y los individuos no solitarios engendran típicamente descendencia, los nodos ancestrales de los árboles arraigados dentro de los anillos matrimoniales pueden ser tanto individuos (hombre, mujer) como parejas, sin violar el 
criterio cíclico simple de los anillos. A través del teorema iii (ramificación) ${ }^{15}$, son posibles al menos dos ramas sin violar el criterio cíclico simple. Como corolario, el nodo ancestral es el único tipo de nodo en un anillo matrimonial que genera ramificaciones. El que la ramificación sea entre hermanos totales, bien sean paternales o maternales, $o$ indeterminada (con una correspondiente pareja parental, padre, madre, o progenitor desconocido), es algo que se hará evidente si las relaciones entre hermanos están convenientemente codificadas desde la base de datos. Entonces, cuando los anillos de los cuatro tipos sean calculados y comparados, los tipos de ramificaciones podrán distinguirse.

\section{TERMINOLOGIA ESTADISTICA DE LOS MATRIMONIOS PREFERENCIALES}

Ciclos cortos. Las reglas de prohibición del incesto - para hermano-hermana, madre-hijo, padre-hija, u otras díadas- prolongan típicamente la longitud mínima del ciclo que puede encontrarse en las redes matrimoniales. Las comunidades difieren en sus reglas proscribiendo el matrimonio con varios tipos cercanos o lejanos de primos, tíos, tías y otros parientes y afines, incluyendo proscripciones tales como no casarse con un WiBrWi, por ejemplo [White, n.d.].

Preferencias. Entre los matrimonios permitidos, esperaríamos encontrar muchas comunidades en las que hay preferencias por los parientes cercanos, es decir, matrimonios que implican una gran preferencia por los ciclos cortos sobre los largos en el matrimonio, una vez excluido el incesto y las proscripciones matrimoniales.

Frecuencias absolutas. Las frecuencias de los matrimonios observados clasificados por tipos pueden proporcionar una indicación de primer orden sobre la preferencia matrimonial, pero la evidencia sobre la preferencia debe ser evaluada cuidadosamente.

Porcentajes. Para cada tipo de pariente que pueda ser tomado en matrimonio (por ejemplo, $\mathrm{FaBrDa}, \mathrm{BrWi}$ ), es también necesario calcular los porcentajes de aquellos parientes que son realmente tomados en matrimonio. Esto puede hacerse con Pajek creando fragmentos para cada tipo de anillo matrimonial, pero

15 Esto puede ser demostrado como sigue. Debido a que cada familia en un ciclo matrimonial tiene un número exacto de encadenamientos matrimoniales y, de esta forma, como mucho dos ramas, siempre y cuando el árbol ancestralmente arraigado para una familia tenga una rama no generada por dos nifios de la raíz, entonces el único camino por el que el antepasado puede implicarse en un ciclo matrimonial es a través de un matrimonio: si ese matrimonio está dentro del mismo árbol familiar, sólo puede haber una rama; si es con otra familia, entonces solamente un nodo de una línea descendente desde el antepasado puede ser el otro matrimonio implicado en el anillo matrimonial y, por ello, ese árbol tiene solamente una rama. El cualquiera de los casos resulta una contradicción, de ahí que el teorema resulte demostrado.

EMPIRIA. Revista de Metodología de Ciencias Sociales. N. ${ }^{\circ}$ 10, julio-diciembre, 2005, pp. 37-69. 
eliminando el vínculo matrimonial mismo, dejando solamente un camino para definir el fragmento (como un anillo nulo) y no un ciclo. Para obtener este porcentaje en la relación $\mathrm{FaBrDa}$, por ejemplo, el número de anillos matrimoniales de $\mathrm{FaBrDa}$ se divide entre el número de caminos de $\mathrm{FaBrDa}$. Estos tipos de porcentajes fueron ya calculados para los parientes consanguíneos en el programa para análisis de p-grafos Par-Calc [White and Jorion 1992, 1996], pero pueden ahora calcularse, usando Pajek, para los parientes políticos.

Distribuciones de frecuencias. Aquí, consideraremos tres métodos para mostrar las distribuciones de frecuencias:

1. Ordenación de los tipos de matrimonio por sus frecuencias o porcentajes, con las frecuencias en el eje $Y$ del plano y la ordenación de estas cantidades (lazos accesibles) en el eje $X$. El uso del cálculo de cohesión circular puede mostrar que sólo se necesita un número limitado de tipos con frecuencia alta (con pocos lazos entre sus frecuencias) para dar cuenta de los ciclos independientes en la red matrimonial.

2. Denominar el eje $\mathrm{X}$ descriptor de frecuencias para los tipos (frequency descriptor for types), empezando con 1 para aquellos tipos con la frecuencia o el porcentaje más bajo subiendo hasta el valor más alto. En este caso el número de tipos que tiene designado este valor se pone sobre el eje Y. De ahí que, si hay 200 tipos con frecuencia 1, el número de matrimonios implicados es $1 \times 200=200$, mientras que si hay 100 tipos con frecuencia 2, representan $200=2 \times 100$ matrimonios. En el extremo habrá muy pocos tipos con alta frecuencia, pero éstos representarán muchos matrimonios. Cuando se use el cálculo de cohesión circular y sólo un cierto número de tipos de frecuencia alta sean suficientes para contabilizar los ciclos independientes, este método dará lugar a discontinuidades en el grafo de valores $\mathrm{x}-\mathrm{y}$. Para buscar relaciones continuas entre los valores $\mathrm{x}$ $y$, podemos usar una medida acumulativa de frecuencias, tal como sigue.

3. Denominar el eje $\mathrm{X}$ frecuencia acumulativa de todos los tipos que tienen sucesivamente menor frecuencia de ocurrencia y el eje $\mathrm{Y}$ número acumulativo de tipos. Este gráfico permite la interpolación continua entre los pares de valores $x, y$ y la consideración de las formas y pendientes de la curva bajo varias transformaciones de los ejes x-y (gráficos lineales, logarítmico sobre un eje, y doblemente logarítmicos)

Cuando las distribuciones se construyen de una de estas tres formas, aunque preferiblemente de la tercera, podemos observar varios tipos de relaciones entre los pares de valores de los ejes $\mathrm{x}-\mathrm{y}$, tal como se indica:

- Relación escalonada discreta. Aquí, en el método 1, algunos de los tipos más frecuentes aparecerán unidos en línea de manera aproximada, posiblemente con un segundo o tercer escalón de líneas de unión. 
- Relación lineal. Uno o varios de los métodos gráficos muestran una relación lineal entre las $\mathrm{X}$ no logarítmicas y los valores de $\mathrm{Y}$. Esto indica la preferencia por «una mayor cantidad» de los ítems más frecuentes, con los ítems menos frecuentes como sustitutos parciales.

- Relación exponencial o logarítmica. Aquí la relación x-y es lineal cuando un eje, pero no ambos, es logarítmico. La interpretación más clara tendrá lugar usando el método 3. Esto no indica necesariamente un orden preferencial porque puede también ocurrir con frecuencias generadas aleatoriamente para tipos diferentes. La cuestión aquí, como con a) y b), es si el orden es predecible por cierta lógica preferencial que pueda ser, a su vez, predicha con antelación a partir de algunas características independientes de los individuos de la comunidad.

- Relación basada en una ley de potencias (power-law relationship). Aquí la relación $x-y$ es lineal cuando ambos ejes son logarítmicos (lo cual es posible únicamente para los primeros $r$ ciclos independientes). La interpretación más clara tendrá lugar cuando se use el método 3. Esto sugiere que hay elementos diferencialmente auto-amplificadores en el ordenamiento. El modelo apropiado debe buscar componentes independientes que se incluyan dentro de la ordenación, cada uno ponderado diferencialmente para producir, tanto la relación de potencias (cuanto más elevado sea el producto o la suma de ponderaciones, mayor será la auto-amplificación) como el ordenamiento mismo.

\section{FORMATO DEL CÁLCULO MATRIMONIAL Y ANÁLISIS}

La definición y los métodos para enumerar los tipos de matrimonio nos permite identificar todos los matrimonios en una red genealógica para cada tipo dado, y estas distribuciones de frecuencia pueden ser analizadas para evidenciar los matrimonios preferenciales. Una vez que la identificación efectiva de los matrimonios por tipo se ha completado, surge una nueva cuestión desde la teoría de la cohesión circular: si eliminamos matrimonios sobre la base de las preferencias que se presumen, contemplándolos como dentro del conjunto de ciclos independientes, ¿qué les ocurre a las frecuencias de los tipos de matrimonio restantes? El teorema i de ciclos independientes requiere que éstos incluyan tipos no-independientes respecto a los tipos preferenciales, y así, estas frecuencias disminuirán. Cuando queden pocos o ningún ciclo tras una serie de eliminaciones, es válido decir que se ha ofrecido un cómputo del conjunto total de ciclos de la red en términos de ciclos preferenciales e independientes.

En el método de extracción de matrimonios propuesto aquí y operacionalizado por Hamberger et al. [2004], se necesita definir un mínimo de cinco re- 
laciones, siendo opcional una sexta ${ }^{16}$. Cinco son primitivas en el sentido de no ser el resultado lógico de ninguna composición de otras, también primitivas (por ejemplo, el hijo del abuelo = el padre del padre). La sexta, la relación entre hermanos, es una relación que no es primitiva, pero se deriva de tener padres comunes. Estas seis relaciones en el formato del cálculo matrimonial son:

$$
\begin{array}{lll}
\text { F-D (arco) } & \text { M-D (arco) } & \text { Matrimonio (arco) } \\
\text { F-S (arco) } & \text { M-S (arco) } & \text { Hermanos (arista) }
\end{array}
$$

Si las cinco relaciones de parentesco primitivas se utilizan para definir fragmentos de búsqueda, usándolos con una red de parentesco codificada en el mismo formato, los fragmentos del anillo pueden ser codificados para incluir antepasados individuales o parejas ancestrales en los componentes del árbol familiar del anillo. Se puede diseñar anillos de tipos complementarios para incluir sólo antepasados femeninos, sólo antepasados masculinos, sólo parejas ancestrales, o inespecíficas. Estas distinciones son importantes para los tipos de ramas de hermanos que aparecen desde los nodos ancestrales.

Un formato reticular alternativo introduce la relación de hermanos y borra el nodo ancestral parental hasta una rama fraternal en el árbol familiar. Esto introduce ciclos en el grafo que no se deben al matrimonio, pero que ocurren dentro de las familias nucleares con dos o más hijos. La fórmula apropiada para el máximo número de ciclos matrimoniales independientes dada más arriba es $r=k-n+1-\Sigma_{k=1, s}\left(n_{s}-1\right)$ donde el subíndice $s$ es el número de grupos de hermanos con dos progenitores y $n_{s}$ es el tamaño de cada uno ${ }^{17}$.

${ }^{16}$ Un p-grafo bipartito constituye una formalización reticular alternativa para este propósito desde el momento en que tiene nodos individuales al igual que nodos matrimoniales, y la eliminación de un matrimonio consiste en reemplazar el nodo matrimonial con líneas descendentes desde los progenitores individuales a los hijos individuales. Sin embargo, cuando esto se hace se crean ciclos que no corresponden a ciclos matrimoniales, de forma que debe aplicarse una variante de la fórmula correctora para los grafos Ore (1960) con el fin de calcular el número de ciclos matrimoniales que queden.

17 Una aproximación de este número se calcula en un grafo Ore borrando aristas de hermanos y reencadenamientos entre esposos, calculando entonces el grado de cada nodo, que se graba como una partición, y calculando después los encadenamientos de hermanos usando Net/Transform/Add/Sibling/Edges/Input. Entonces, usando Net/Transform/Remove/all Arcs, todos los arcos son borrados. Ahora la partición previamente grabada, posicionada ya en la ventana de partición, se usa para hacer Operation/Extract -seleccionanado Network/Partition/values from l up-, y Net/Partition/Degree/Input degree es usada para calcular el número de encadenamientos de hermanos en las aristas. Finalmente, Operation/Extract desde Network/Partition/values from 1 up producirá otra vez un cómputo de hermanos para cada individuo de la partición. Estos conjuntos de hermanos deben calcularse para cada tamaño de grupo, etiquetándolos como $k=1$ para $(s-1)$, donde $s$ es el mayor número de hermanos, y añadiendo los productos de cada frecuencia por su índice $k$. La razón por la que esto es aproximado es que puede haber medio-hermanos en la red.

EMPIRIA. Revista de Metodología de Ciencias Sociales. N. ${ }^{\circ}$ 10, julio-diciembre, 2005, pp. 37-69.

ISSN: 1139-5737 


\subsection{Métodos y medidas}

Muestras. Cientos de muestras genealógicas codificadas se encuentran disponibles para el análisis [White, Houseman, Schweizer, 1993] y miles de antropólogos han recopilado genealogías completas para las comunidades que han estudiado. El Proyecto TIME (ver [Hamberger et al., 2004]) ha transformado muchas de estas y otras redes genealógicas a formato de cálculo matrimonial.

Software. Desde el primer año de su implementación en 1996, el programa Pajek para grandes análisis de redes ha proporcionado y tiene, desde su actualización, una serie de algoritmos para las redes de parentesco y las genealogías [Batagelj, Mrvar 1998, 2002, 2004; White, Batagelj, Mrvar, 1999]. El índice de reencadenamiento, una medida del punto en el que un grafo con $\mathrm{k}$ antepasados independientes alcanza el máximo número posible de reencadenamientos, fue implementado en 1998. Una versión definitiva de Pajek $(1.01,2004)$, acompañado de un manual de instrucciones [de Nooy, Mrvar, Batgelj, 2004], proporciona formato y método en el estudio de la cohesión circular para los datos del cálculo matrimonial. Los comandos Macro para el análisis del parentesco vienen con la instalación de Pajek.

Fragmentos. La búsqueda e identificación de fragmentos en los grafos fue implementada en Pajek en 1997. En 2003, desarrollé un conjunto de fragmentos de parentesco para identificar diferentes tipos de matrimonios consanguíneos en los p-grafos. El recuento de fragmentos de Pajek elimina isomorfismos (por ejemplo, dos hermanos que se casan con dos hermanas se cuenta como un único fragmento), lo que permite contar los tipos de matrimonios por el número de subgrafos o por el número de matrimonios. Por ejemplo, los anillos HuSiHi tienen transformaciones isomorfas entre los dos matrimonios implicados en el anillo. Sabiendo el número de isomorfismos de cada grafo, por ejemplo los dos isomorfismos para el intercambio de hermanas, los cómputos de fragmentos son fácilmente convertidos en cómputos de frecuencias para los individuos. Hamberger et al. [2004] desarrolla un conjunto mejorado de fragmentos de parentesco en el formato del cálculo matrimonial para identificar diferentes tipos de reencadenamientos, incluyendo los matrimonios de sangre. Esta aproximación usa el software de Jürgen Pfeffer (FAS-Research, Vienna) para convertir un archivo de parentesco con formato Excel (Ego, Sex, Fa, Mo, Spouse) en un archivo genealógico Pajek en el nuevo formato. Los vínculos entre hermanos deben ser entonces añadidos y los bucles resultantes eliminados, siendo éstas operaciones simples para Pajek ${ }^{18}$. Para encontrar tipos de matrimonios como frag-

${ }^{18} \mathrm{El}$ procedimiento consiste en hacer un archivo *.xls file con Ego\#, Name, Sex $(\mathrm{H}=$ hombre, $\mathrm{F}=$ mujer), Fa\#, Mo\#, Spouse\#, clasificado por sexo, borrar todos los números de esposos para los hombres (para hacer que la relación de esposos sea dirigida), ejecutar entonces el programa Gen2Pajek para crear un archivo Pajek*. net. Este archivo requiere la adición de encadenamientos de hermanos, lo cual es una operación Net/Transform/Add (opción Input para la equivalencia de hermanos) en Pajek.

EMPIRIA. Revista de Metodología de Ciencias Sociales. N. ${ }^{\circ}$ 10, julio-diciembre, 2005, pp. 37-69.

ISSN: 1139-5737 
mentos en la red, el archivo genealógico es analizado para tipos de fragmentos sucesivos, lo cual es ahora una opción standard en Pajek ${ }^{19}$. Los fragmentos encontrados de un cierto tipo pueden ser conservados sin reenumerar nodos, de forma que los matrimonios de un tipo dado puedan ser sustraídos de una red dada $^{20}$. Esta característica se usa para el método de extracción de matrimonios en el análisis de la cohesion circular ${ }^{21}$.

\subsection{Hipótesis}

White y Houseman [2002, p. 78-79] postularon una tricotomía de tipos de comunidad respecto a las fuentes de la cohesión marital:

- H1. Las comunidades con muchos matrimonios consanguíneos pueden tener preferencias que siguen una ley de potencias en los matrimonios de sangre, pero serán neutrales en los reencadenamientos multifamiliares, es decir, con diferencias que están exponencialmente distribuidas. El modelo de cohesión circular apropiado para examinar aquí es el que considera que los ciclos independientes que generan endogamia estructural son los matrimonios de sangre.

- H2. Las comunidades con pocos matrimonios consanguíneos pueden tener preferencias que siguen una ley de potencias sobre los reencadenamientos multifamiliares. Éstas pueden mostrar uno de estos dos subtipos:

Preferencias con ley de potencias en reencadenamientos de dos familias Preferencias con ley de potencias en reencadenamientos de tres familias

19 El procedimiento consiste en leer los fragmentos como un archivo *.paj (File/Pajek Project file/Read), y cargar entonces el archivo genealógico hecho con Gen2Pajek, usando la opción Nets/Second network para cargarlo con el fin de hacer comparaciones. La ventana de redes se pincha para seleccionar de nuevo el primer fragmento, y la opción Nets/First network se usa para permitir que el fragmento (primera red) sea encontrado en la red (segunda) más amplia usando la opción Nets/Find (1 en 2). Las opciones deben ser establecidas en Nets/Find, antes de este último paso, para comprobar $[\mathrm{x}]$ valores de líneas, $[\mathrm{x}]$ Extraer subred y $[\mathrm{x}]$ Conservar todos los vértices tras la extracción. Ello permitirá encontrar los matrimonios que serán sustraídos desde la genealogía. Si se buscan los anillos, entonces la opción debe ser comprobada para [x] subgrafos inducido. De otra forma, si [ ] un subgrafo inducido se deja sin comprobar, entonces el ciclo en el fragmento será encontrado independientemente de que el subgrafo que se ha identificado como aquel que contiene este ciclo, tenga o no tenga encadenamientos adicionales que no estén en el fragmento.

${ }^{20}$ Debido a que los matrimonios tiene el valor líneal 1 , sólo se necesita en la subred extraída usar la opcion Net/Transform/Remove/lines with value/ more than [1] y Net/Transform/Remove/all edges para crear un archivo de red exclusivamente con los encadenamientos matrimoniales. Éste es entonces sustraído cargando el archivo genealogico con la opción Nets/First network y cargando el archivo de matrimonios para que sea sustraído con Nets/Second network, de forma que Nets/difference sustraiga los matrimonios de la red.

${ }^{21}$ Hamberger [Hamberger et al., 2004] desarrolló una serie adicional de macros Pajek para calcular desde este archivo un conjunto tri-modal de datos con individuos, matrimonios y tipos de matrimonios.

EMPIRIA. Revista de Metodología de Ciencias Sociales. N. ${ }^{\circ}$ 10, julio-diciembre, 2005, pp. 37-69. 
Principio de la partición de independencia (cohesión circular). La teoría de la cohesión circular ayuda a explicar las bases de las hipótesis 1 y 2 de White y Houseman [2002]. Cuando la unicidad de los padres y las restricciones contra el matrimonio entre hermanos se aplica para una base genealógica de datos dada, conviene pensar tanto en el conjunto entero de matrimonios consanguíneos que constituyen el grueso de un conjunto S de ciclos independientes, siendo los matrimonios no consanguíneos los que constituyen el grueso del conjunto complementario de ciclos no-independientes, como en el caso contrario. Esto se debe a que entre el conjunto de matrimonios consanguíneos, para cualquier subgrafo inducido con $r=2$ ciclos independientes, encontrado durante la búsqueda de fragmentos, no puede haber ciclos no-independientes ${ }^{22}$, y lo mismo es cierto para el conjunto de matrimonios no consanguíneos. La ausencia de independencia para $r \geq 3$ subgrafos inducidos implicará combinaciones de reencadenamientos consanguíneos y no consanguíneos: dos matrimonios MoBrDa dan lugar a dos matrimonios FaBrDa, como en la Figura 1(b), por ejemplo, sólo si hay intercambio de hermanas en la primera generación. En general, las mayores restricciones en los tipos de reencadenamiento que pueden darse a la vez tienen lugar dentro de las clases de reencadenamiento consanguíneo y no consanguíneo. Los casos complejos, tales como los del ejemplo 1(b) podrían implicar combinaciones de un tipo no consanguíneo y de dos tipos consanguíneos, o viceversa.

Lo que implican conjuntamente el teorema i de ciclo independiente y el principio de partición de independencia es que, si una comunidad tiene preferencias orientadas hacia tipos de parientes consanguíneos, una distribución circular preferencial (preferential ring distribution) de matrimonios consanguíneos implicará normalmente una distribución exponencial (no preferencial) de ciclos matrimoniales reencadenados no-independientes. Lo opuesto se aplicará si una comunidad tiene preferencias orientadas hacia tipos de reencadenamientos no consanguíneos. Normalmente cabría esperar que la distribución de ciclos no-independientes no fuera preferencial porque éstos no se forman independiente-

${ }^{22}$ En el p-grafo que se muestra a continuación, por ejemplo, hay tres ciclos independientes, y mientras la adición de grafos para los dos que comparten una arista formaría un anillo matrimonial MoMoBrDaDa, no se encontraría que este anillo fuera un fragmento de un anillo del grafo, debido a que se requiere que la búsqueda sea para un ciclo simple, no para un par de ciclos.

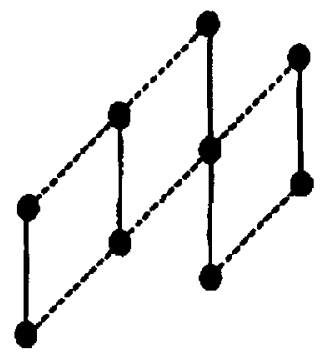

EMPIRIA. Revista de Metodología de Ciencias Sociales. N. ${ }^{\circ}$ 10, julio-diciembre, 2005, pp. 37-69. ISSN: 1139-5737 
mente. Esto proporciona una tercera hipótesis como derivación de la teoría de la cohesión circular.

- H3. Pocas comunidades tendrán preferencias que sigan una ley de potencias en ambos reencadenamientos, tanto consaguíneos como no consanguíneos.

Puede que sea poco probable, aunque no imposible, sin embargo, que ambas distribuciones pudieran ser optimizadas y estructuradas preferencialmente. Esto conllevaría un segundo orden de concatenación de anillos sistemático y preferencial en estructuras cohesivas mayores.

Las hipótesis (H1 y H2) de White y Houseman [2002, p. 78] fueron inicialmente el informe de un modelo empírico de los resultados de una investigación acerca de las redes de parentesco consideradas de forma general. La teoría de la cohesión circular identifica la derivación de estas hipótesis desde los principios reticulares y matemáticos que sostienen los sistemas generales de parentesco. $\mathrm{H} 3$ se sostiene porque el parentesco tiene un componente hereditario (ascendencia) y un componente electivo (afinidad). Las redes biológicas y de otros tipos tienen también contrastes internos de este tipo para ciertos subtipos de relaciones reticulares, de forma que sería esperable una generalización más amplia de $\mathrm{H} 3$ hacia otras ciencias desde los primeros principios. De forma más general:

- H4: Sea cual sea el método para detectar preferencias matrimoniales, habrá algunas tendencias estadísticas para las preferencias de tipo consanguíneo que permitan reducir aquéllas con reencadenamientos no consanguíneos, y viceversa.

Esto toma en cuenta la posibilidad de que una ordenación preferencial de un pequeño subconjunto de matrimonios consanguíneos no descarte preferencias matrimoniales adicionales, y viceversa. Sólo cuando una ordenación preferencial se extiende sobre un rango mayor de tipos matrimoniales, $\mathrm{H} 3$ podría ser operativa.

\subsection{Resultados ilustrativos para el análisis de redes de parentesco}

El enfoque de la cohesión circular se ejemplifica aquí con los datos de los nómadas turcos Ayd1nl1 estudiados por White y Johansen [2004; Johansen, White, 2002]. Los resultados de las siguientes tablas y figuras son ilustrativas de las pruebas realizadas sobre las hipótesis acerca de los nómadas Turcos, con el sistema árabe de parentesco y los derechos hacia el matrimonio FaBrDa. 


\section{Preferencias}

Se considerará primero la evidencia para considerar los matrimonios consanguíneos como preferenciales y, por tanto, como conjunto apropiado de ciclos independientes de la red. Si ambas condiciones fueran ciertas, es posible que los reencadenamientos matrimoniales, considerados como ciclos no independientes, no requieran una explicación más amplia en términos de preferencias. Las evidencias que prueban que los matrimonios consanguíneos son preferenciales (y que los reencadenamientos no consanguíneos no lo son) vienen de White y Johansen [2004, p. 275-278], como se muestra en las figuras, algunas de las cuales son discutidas en White y Houseman [2002].

La Figura 4 utiliza el método gráfico 2 para explotar el gradiente de frecuencias para todos los tipos de matrimonios consanguíneos, sin tener en cuenta la distancia del parentesco ${ }^{23}$. Aquí, el eje $x$ es una variable del número de esposos cuyo matrimonio encaje en uno de los 234 tipos de parentesco sanguíneo dentro del rango de los quintos primos ( 7 generaciones a partir de un ancestro común). El eje y es el número de tales tipos de matrimonio con exactamente el número $x$ de esposos relacionados.

Desde el gráfico podemos ver que 156 de los 234 tipos de matrimonios tienen una frecuencia matrimonial de uno en el conjunto de datos. Este número cae hasta 36 de los 234 para los que tienen dos matrimonios. Si el gráfico fuera exponencial, dicho número seguiría cayendo en una fracción constante, como desde 156 hasta 36 , o como de 10 a $~ 2$ y rápidamente a cero. En su lugar el gráfico sigue una ley de potencias, y cae desde 156 hasta 36 , hasta $\sim 18$, hasta $\sim 10$, hasta $\sim 5$, mostrando la cola extendida de una distribución basada en una ley de potencias. La larga cola indica que pocos tipos tienen frecuencias mucho más altas que las que tendrían lugar si los tipos de matrimonios compañeros fueran escogidos aleatoriamente. El outlier extremo en esta explotación de frecuencias por tipo es el matrimonio FaBrDa, para el cual hay 32 casos, casi el doble del siguiente matrimonio más frecuente, del tipo MoBrDa. La distribución tiene un exponente fijo ca. 2 , una ley de potencias inversamente cuadrada cuya ecuación es aproximadamente $y=156 / x^{2}$, siendo $y$ el número de tipos cuya frecuencia $x$ se encuentra entre 1 y 32 . El ajuste de la curva a los datos es $r^{2}=0,83$ con una pendiente estimada de 1,97. Debido a que no se espera que la distribución en base a una ley de potencias aparezca aleatoriamente, éste será un indicador de la distribución preferencial. Leyes de potencias de esta clase sugieren redes que operan como sistemas autoorganizados con propiedades fractales, donde las frecuencias de comportamientos generadores de cohesión son auto-escalares, por ejemplo, con los diámetros de los ciclos cohesivos formados. Una conducta similar en diferentes escalas tiende a agotarse, de forma que cuanto más difusa sea

${ }^{23}$ La distancia del parentesco puede ser calculada de varias formas, una de las cuales es el camino más corto desde el individuo A hasta B en el formato del cálculo matrimonial del parentesco. Otras medidas son más específicas culturalmente.

EMPIRIA. Revista de Metodología de Ciencias Sociales. N. ${ }^{\circ}$ 10, julio-diciembre, 2005, pp. 37-69. 


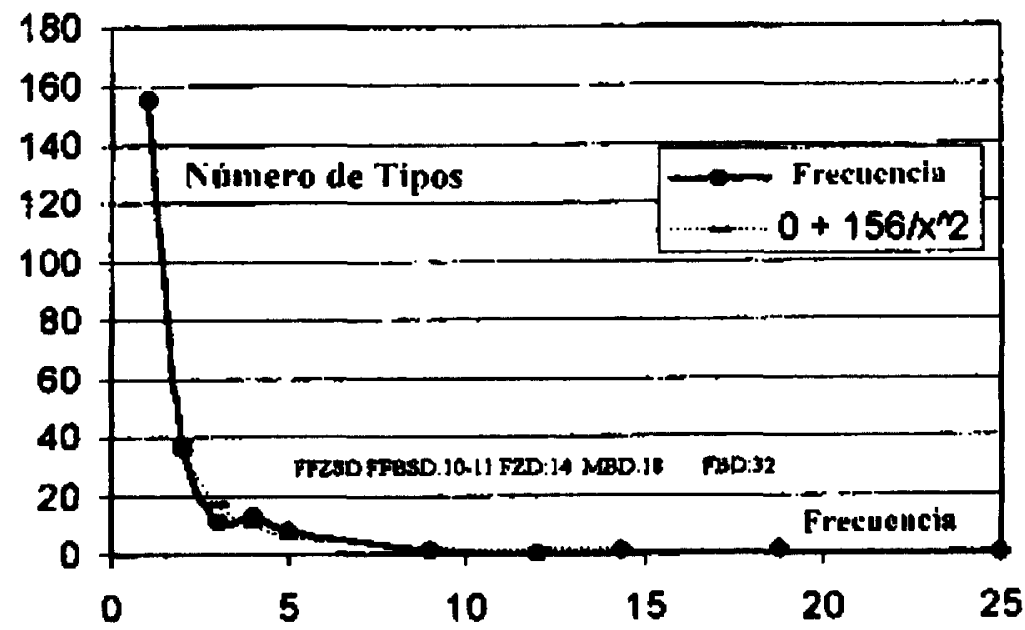

Figura 4. Fractalidad según la ley de potencias de las Frecuencias Matrimoniales.

la forma de los reencadenamientos maritales, más baja será su frecuencia. Los tipos de matrimonio Aydınlı con las mayores frecuencias (FaBrDa, MoBrDa, Fa$\mathrm{SiDa}$ también tienden a seguir una ordenación de frecuencias que refleja la distancia del parentesco vista desde una perspectiva patrilineal. Para los ciclos matrimoniales de mayor longitud, la ordenación de frecuencias tiende a escalarse fractalmente de acuerdo al nivel de solidaridad corporativa patrilineal escalada por la distancia hasta el antepasado común.

La Figura 5 repite el análisis de la Figura 4, registrando esta vez los valores en los ejes $x$ e $y$, y ajustando una línea recta en el plano. El ajuste se aproxima a una distribución según una ley de potencias. La terminología estadística de esta relación se discute tras esta introducción en la sección 4 - la hipótesis relativa a los elementos diferencialmente autoamplificados en la ordenación. El proceso de amplificación resultante de estas frecuencias es mayor cuanto más estrecha sea la distancia de unión, cuando los vínculos masculinos sean ponderados de forma más elevada que los vínculos femeninos, de forma que $\mathrm{FaBrDa}$ resultará el más alto de la ordenación.

Los modelos de matrimonio fractal funcionan más bien como los lazos fuertes y débiles de Granovetter [1973]. Los lazos más fuertes y más frecuentes (de muchos menos tipos) trabajan en distancias más estrechas, en este caso orientadas concéntricamente hacia los parientes cercanos y patrilineales, mientras que los lazos más débiles de cada tipo son individualmente menos frecuentes, aunque funcionen como un conjunto de forma distribuida sobre distancias más largas. A diferencia de la forma en que las preferencias matrimoniales son formuladas usualmente, como reglas discretas, el modelo fractal es continuadamente escalado, más que una simple dicotomía de tipos preferidos de matrimonio. 


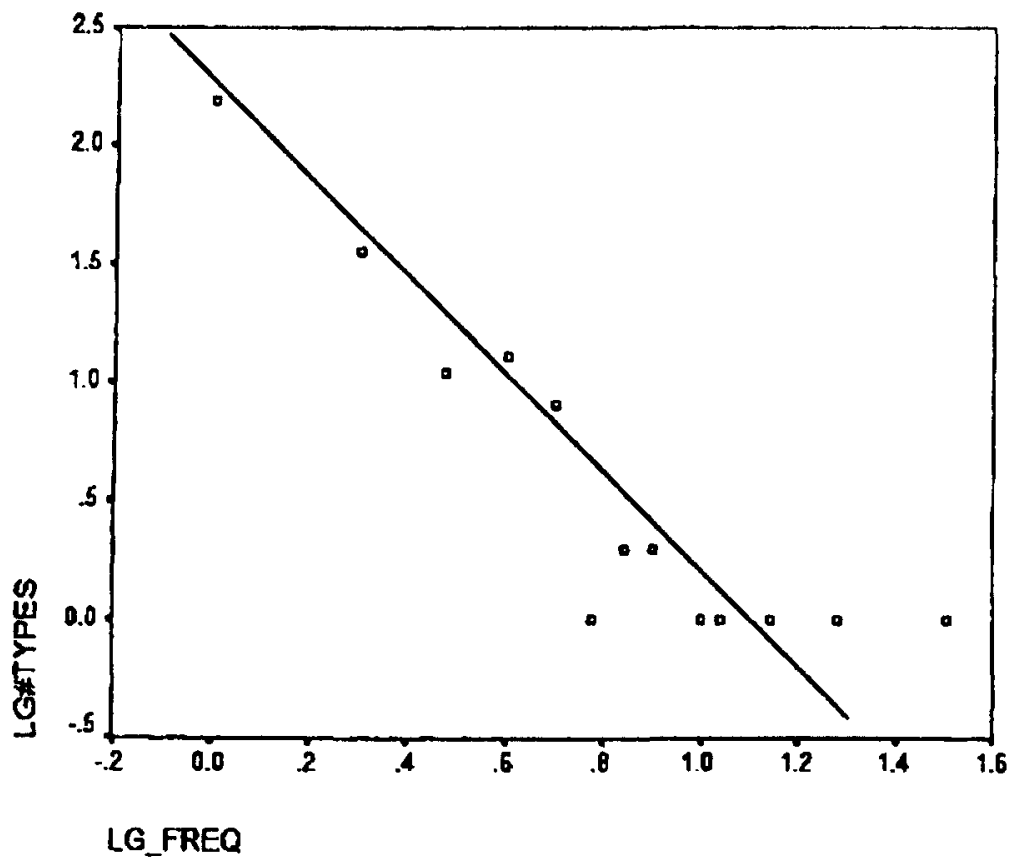

Figura 5. Ley de potencias para Frecuencias de Matrimonio- doblemente logarítmica para la Figura 4, con línea ajustada y pendiente $\sim 2$.

Estos resultados pueden compararse con los del método gráfico de 4.1, como se muestra en la Figura 6. Aquí, la línea de frecuencias absolutas muestra el resultado de la representación de las frecuencias de todos los 234 tipos de matrimonios consanguíneos hasta los primos de orden séptimo, ordenados gradualmente con frecuencias logarítmicas sobre el eje $y$, y con el número logarítmico de observaciones para este tipo y frecuencia sobre el eje $x$. La distribución de las frecuencias absolutas es lineal en el gráfico doblemente logarítmico y, de esta forma, se aproxima a una distribución según una ley de potencias, ajustando nuestra observación global sobre el modelo de matrimonio fractal. El resto de aspectos de las distribuciones de matrimonio consanguíneo representados por este método, son el número de posibles esposos de cada tipo en la parte superior de la Figura 6 y el porcentaje de casados de cada tipo en la parte inferior.

La curva de frecuencias de tipos posibles de esposos de la parte superior (todos los disponibles en una categoría dada) muestra un descenso exponencial o una distribución logarítmica. Aquí, $\mathrm{FaBrDa}$ es el tipo de pariente disponible más frecuente, y MoBrDa el siguiente. La curva para el porcentaje de casados de cada tipo dentro de los disponibles, es también una disribución logarítmica, a diferencia de la Figura 4, de nuevo con $\mathrm{FaBrDa}$ como el porcentaje más alto y MoBrDa el siguiente. La forma logarítmica se debe al hecho de que hay muchos 
menos tipos de consanguíneos en cada distancia de parentesco conforme nos desplazamos más cerca del ego (cuatro tipos de primeros primos). Igualmente, en una red limitada, conforme nos desplazamos hacia relaciones muy distantes, éstos disminuyen si hay pocos antepasados apicales. Ello se debe a que una gran cantidad del vasto número de posibilidades combinatorias no se producen, y las relaciones más próximas ya han utilizado muchos de los parientes de la red. Solamente las frecuencias absolutas ajustan en la distribución según la ley de potencias característica de fractalidad. Así es como debería ser en un sistema que está conductualmente autoorganizado. En esta sociedad, FaBrDa no es únicamente un matrimonio preferido, sino un pariente cuya proximidad es más probable debido a la preferencia de que los hermanos y sus hijos vivan juntos, permanezcan juntos y trabajen juntos.
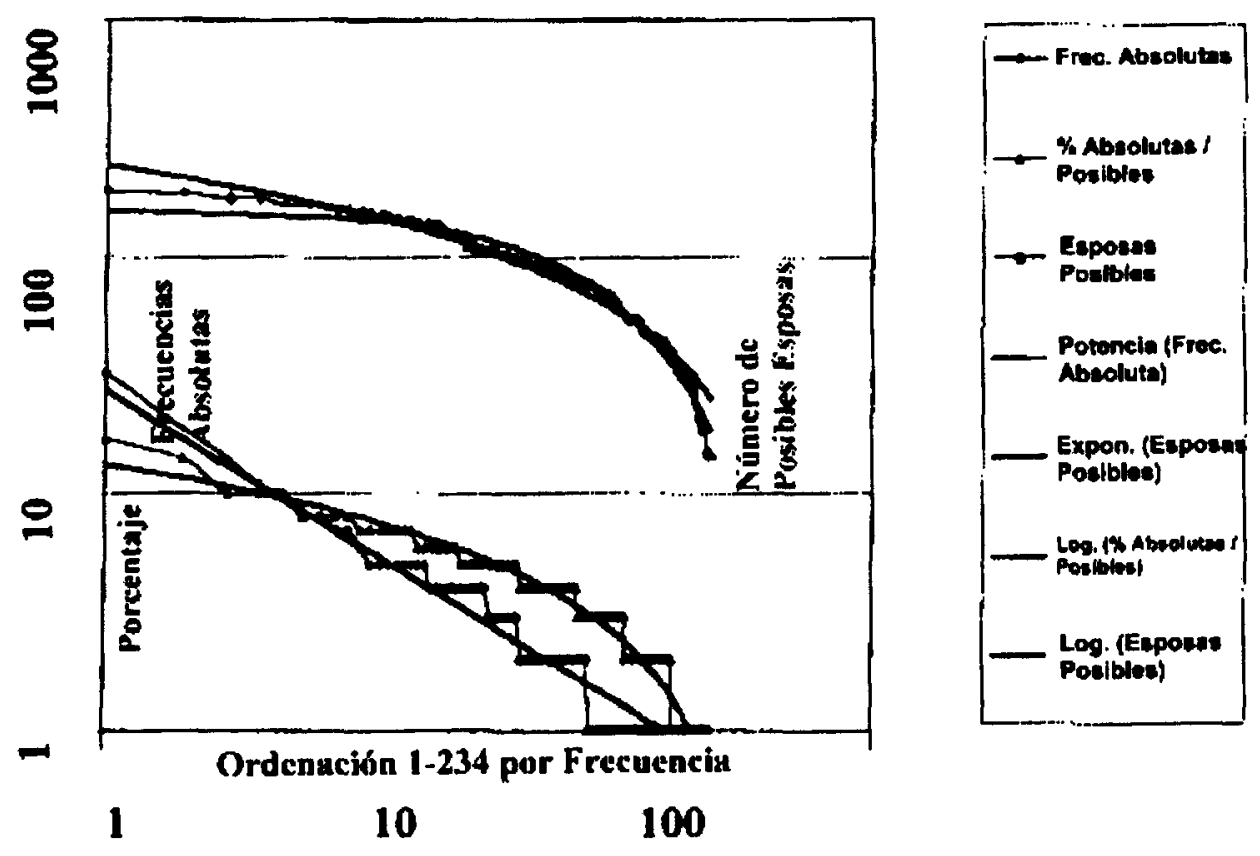

Figura 6. La Fractalidad de las Frecuencias de Matrimonios Consanguineos.

Los análisis de matrimonios reencadenados no-consanguíneos para el caso del clan nómada de los Aydınl1, comparables a los de matrimonios consanguíneos, no muestran tendencias que sigan una ley de potencias, lo cual es consistente con $\mathrm{H} 1$ y $\mathrm{H} 3$. 


\section{Resultados de la Cohesión Circular para los Nómadas Turcos Aydınlı}

Los resultados de la cohesión circular en las distribuciones preferenciales para el clan nómada muestran que, aunque el matrimonio consanguíneo más frecuente es FaBrDa, estos matrimonios no son esenciales para la cohesión del clan. Es la clase mayor de matrimonios consanguíneos la que genera esta cohesión; la hipótesis es que constituyen el conjunto de ciclos que deberíamos considerar como independientes. Esto se establece en la hip6tesis 5 y es comprobado en la Tabla 1.

- H5 (Aydınlı): La eliminación del matrimonio FaBrDa no reduce la endogamia estructural, pero la endogamia estructural está generada por la clase mayor de matrimonios consanguíneos.

Para los datos de la Tabla 1, el bicomponente estructuralmente endogámico se calculó antes de añadir encadenamientos fraternales como aristas. El tamaño del bicomponente es 960 . El cálculo de la cohesión circular se usó para hacer la Tabla 2, encontrando matrimonios $\mathrm{FaBrDa}$ en la misma base de datos, borrando solamente los encadenamientos del matrimonio $\mathrm{FaBrDa}$, y conservando el resto del grafo usado para la Tabla 1. El bicomponente seguidamente se calcula de nuevo, pero su tamaño sigue siendo 960. Los matrimonios Aydınlı del tipo $\mathrm{FaBrDa}$ tienen lugar, entonces, entre parientes vinculados cohesivamente independientemente de sus relaciones como $\mathrm{FaBrDa} / \mathrm{FaBrSo}$.

Tabla 1

\begin{tabular}{lcl}
\hline nodos en bicomponente $=960$ & Arcos & Aristas \\
\cline { 1 - 2 } Número de líneas con valor $=1$ & 248 & \\
2573 (p-tur-bico) \\
Número de líneas con valor \# 1 & 1749 & 0
\end{tabular}

Tabla 2

FaBrDa: 34 matrimonios encontrados y borrados

Después de la extracción de FaBrDa y de las aristas fraternas:

\begin{tabular}{lcl}
\hline nodos en bicomponente $=960$ & Arcos & Aristas \\
\cline { 1 - 2 } Número de líneas con valor $=1$ & 214 & \\
Número de líneas con valor \# 1 & 1749 & 0
\end{tabular}

La Tabla 3 muestra los cálculos de los ciclos independientes del grafo matrimonial para los matrimonios de primos cercanos comparados con la red genealogica total. Hay 116 ciclos independientes que quedan explicados por estos matrimonios consanguíneos de un total de 266, quedando 150 sin explicar, 
como también se sugiere, examinando la Figura 4, para los matrimonios consanguíneos con frecuencias de uno o dos por tipo. Entonces, aunque el primer y segundo matrimonio entre primos ocurren entre linajes patrilineales, tampoco consiguen dar cuenta de la cohesión del clan Aydınlı.

Tabla 3

Ciclos Independientes en el Matrimonio entre Primos

Grafo (primos de primer y segundo orden) comparados con el grafo Total

En el grafo de fusión de primos

Número de líneas

$315-(\sin$ aristas)

Número de nodos

176

$139-11-11$ ciclos independientes $=116$

En el grafo total

Número de líneas

1997 (sin aristas)

Número de nodos

960

$1037-1-771$ ciclos independientes $=266$

diferencia

150 sin explicar

¿Qué ocurre si todos los matrimonios consanguíneos son eliminados?

- H6 (Ayd1nl1): La eliminación de todos los matrimonios consanguíneos elimina también casi todos los demás reencadenamientos de dos familias $\mathrm{y}$ otros reencadenamientos matrimoniales.

H6 se sostiene para todos los reencadenamientos matrimoniales sistemáticos excepto para 11 matrimonios entre pares de hermanos: una vez que los matrimonios consanguíneos son eliminados, los reencadenamientos fraternos (intercambios de hermanas y matrimonios $\mathrm{BrWiSi}$ ) disminuyen desde una frecuencia de 135 hasta 11, y ninguno de los restantes reencadenamientos de dos familias tienen una frecuencia significativa.

El cálculo de la cohesión circular, en el ilustrativo caso de los nómadas turcos, es consistente con la consideración de la clase de matrimonios consanguíneos como fuente de reencadenamiento preferencial entre esposos, y explica casi completamente la endogamia estructural de la red sin tener que tomar en cuenta el reencadenamiento multifamiliar excepto para algunos de los matrimonios entre pares de hermanos. El matrimonio FaBrDa per se no explica nada de la endogamia estructural, lo cual se debe casi completamente a la variedad de otros matrimonios consanguíneos, la mayor parte de los cuales se producen entre linajes. En este caso, estos resultados hablan del hecho de la presencia de dos polos principales en la estructura matrimonial, uno ampliamente distribuido entre las opciones de matrimonio consanguíneo dentro del clan, muchas de ellas con 
consanguíneos distantes. El otro es el hecho de que los matrimonios cerrados que refuerzan la cohesión del linaje, tales como el $\mathrm{FaBrDa}$, se dan fácilmente con la alta frecuencia de mujeres co-residentes disponibles, y ello explica también el porcentaje más alto de matrimonios con éstas en cualquier tipo dado de matrimonio. Dado que el número de ciclos matrimoniales consanguíneos es suficiente para explicar casi todos los ciclos de matrimonio independientes, los encadenamientos sucesivos no consanguíneos pueden ser considerados para la mayor parte como ciclos no independientes y productos derivados de la concatenación de matrimonios consanguíneos, como se ejemplifica en la Figura 1. Sin embargo, debido a que hay alguna evidencia independiente para los matrimonios independientemente cohesivos entre pares de hermanos (las 11 excepciones de H6) y el número muy elevado de matrimonios consanguíneos concatenados que producen tales matrimonios, como los de la Figura 1(b), hay una buena razón para considerar que estas concatenaciones, como estructuras de cohesión de segundo orden, son también preferenciales entre los Aydınlı.

\section{CONCLUSIONES}

El término cohesión circular (ring cohesion) se usa para transmitir la posibilidad de encontrar las conexiones micro-macro entre la conducta individual que genera ciclos elementales en las redes, y el mayor nivel de agrupamientos cohesivos creados en una red. La primera crea la segunda, y la segunda (la cohesión estructural de los grupos) tiene importantes consecuencias sociopolíticas, económicas y de otros tipos que incluyen la creación del contexto para las elecciones miroconductuales. La estructura interna y la extensión de las redes cohesivas en el nivel macro son parte de lo que da a las comunidades y a los grupos sociales su estructura y dinámica distintivas. Para entender explícitamente cómo estos linajes micro-macro funcionan a través de diferentes comunidades y estructuras sociales, pueden finalmente llegar a formar parte de nuevas teorías fundacionales relacionadas con la organización, estructura y dinámicas sociales.

Para el análisis general de redes, la teoría de la cohesión circular proporciona una solución a los problemas del estudio de la formación cíclica y la relación entre la cohesión local a través de la conducta, que conduce a los ciclos relacionales y a propiedades más globales de la cohesión reticular. Para el análisis de las redes matrimoniales en particular, la teoría de la cohesión circular proporciona una solución a los problemas que han plagado este campo desde hace tiempo [Schneider, 1965]: ¿cómo contar los ciclos? ¿cómo contribuyen los ciclos matrimoniales a la cohesión social? ¿cómo medir la cohesión en una red genealógica? ¿cómo definir las fronteras de una comunidad cohesiva? ¿cómo determinar los matrimonios preferenciales? ¿cómo y hasta qué punto los matrimonios preferenciales contribuyen a la estructura endogámica? ¿cúal es la relación entre la cohesión social y la estructura endogámica? La teoría de la cohesión predictiva, tal como fue formulada y comprobada por Moody y White (2003), por ejemplo,

EMPIRIA. Revista de Metodología de Ciencias Sociales. N. ${ }^{\circ} 10$, julio-diciembre, 2005, pp. 37-69. 
responde a algunas de las restantes cuestiones, como: ¿qué ocurre entonces? ¿por qué es importante la cohesión estructural en primer lugar? ¿cuáles son sus consecuencias predecibles? La teoría de la cohesión circular ofrece una extensión importante a la teoría de la cohesión predictiva, vinculando la conducta individual micro a la estructura y dinámicas macro.

El programa Pajek para el análisis de redes, equipado ahora con nuestras sugerencias para el análisis de la cohesión circular, proporciona procedimientos operativos para tratar estas cuestiones, algunas de las cuales han sido ejemplificadas y examinadas en el ejemplo de los nómadas turcos del tipo de parentesco y sistema matrimonial arábicos. En este caso es evidente un gradiente de matrimonio preferencial para el reencadenamiento consanguíneo, pero no para el no consanguíneo. Aislando los matrimonios consanguíneos como un conjunto de matrimonios lo suficientemente grande como para constituir el conjunto independiente total de ciclos matrimoniales, se obtiene un resultado confirmatorio en el que los ciclos reencadenados no consanguíneos son también aislados, de forma consistente con la teoría de la cohesión circular. La teoría es útil, entonces, para delimitar los problemas de explicación y predicción en el desarrollo de la teoría social de redes matrimoniales, y puede ser fácilmente extensible a las redes en general para estudiar las fuentes de la cohesión.

Como teoría más general aplicada a las redes sociales, el problema de la cohesión circular es uno de los gradientes conductuales: ¿cuáles son los contextos locales en los que los individuos hacen elecciones que afectan a las cuestiones más amplias de la cohesión social a través de la formación de ciclos en redes? Este es un problema micro-macro: las elecciones realizadas localmente en una red para formar vínculos pueden, tanto formar, como no formar ciclos. Los pares de ciclos, cuando son concatenados, forman indirectamente ciclos adicionales, fuera de la visión o de la intención del actor local, algunas veces estratégicamente, pero normalmente no como un resultado directo de la acción local. Lo que la cohesión circular ofrece es un conjunto de medidas y teoremas que permite establecer hipótesis sobre conjuntos de ciclos independientes para cualquier red dada y enfrentarlos a la conducta real que los genera, mientras que el conjunto de ciclos no-independientes (típicamente más largos) que necesariamente tienen lugar en la red más amplia, son un producto derivado de esa conducta, aunque la forma en que un conjunto de ciclos independientes simples concatena dentro de la estructura de ciclos no independientes de segundo orden, sea una cuestión adicional, formalizada y analizada en varios ejemplos etnográficos por Hamberger et al. [2004] para las redes sociales.

Los ciclos no-independientes, sin embargo, al estar formados «de espaldas» a los actores locales, son igualmente una parte de las estructuras cohesivas más amplias encajando a los actores y subgrupos en la red. Estas estructuras macro más grandes y la concatenación de ciclos no-independientes puede requerir una comprobación estadística adicional y una explicación si las concatenaciones no son aleatorias, dada la estructura de los fragmentos de preferencias no-independientes. No pueden ser analizadas con el mismo marco estadístico que los ci- 
clos de primer orden, y es necesario analizarlas con un paquete especial para análisis de segundo orden. Teniendo en cuenta ambos, puede requerirse conjuntamente la construcción de una red tanto de primer como de segundo orden para estudiar el linaje micro-macro.

Excepto en el caso de circunstancias inusuales, sin embargo, es hacia el análisis del conjunto independiente de ciclos de menor longitud, hacia donde debe ser enfocado el análisis de la formación de ciclos de primer orden [Vismara, 1997; Batagelj y Zaversnik, 2003]. Qué conjunto específico de ciclos debería considerarse independiente, es una cuestión que consiste en identificar la acción social preferencial o con inclinación dirigida hacia los bloques de edificios más simples de la estructura social. Sólo entonces podremos considerar las cuestiones de segundo orden: ¿cuáles son los caminos, estrategias y beneficios de combinar estos bloques de edificios, si existe alguno más allá de las concatenaciones aleatorias?

La hipótesis que guía la cohesión circular considera que los ciclos cohesivos se forman como resultado de la acción local. En el caso general, debería haber anillos fácilmente reconocibles y localizados, dentro de las percepciones de los actores sociales (ver [Brudner, White, 1997]). Estos contextos localmente definibles son los primeros lugares desde donde se debería mirar la conducta con gradiente dirigido o la conducta preferencial. Adicionalmente, para individuos excepcionales o procesos que impliquen explotar grandes agujeros estructurales en redes [Burt, 1995] para formar ciclos largos pero simples, se podría buscar conducta intencional en el diámetro más largo de los anillos de cohesión que, por otra parte, son típicamente no-independientes de los anillos más pequeños. Es la diferenciación dentro/fuera de los anillos la que ayuda a entender cómo la historia - en el sentido de los efectos de los agrupamientos cohesivos- está hecha tras la espalda de los actores individuales, como resultado de los encadenamientos micro-macro de primer orden, aunque de nuevo, a veces estratégicamente. Las ideas de reglas y estrategias pueden así ser asimiladas al mismo análisis.

Agradecimientos. La financiación parcial para este proyecto fue concedida por ISCOM, Information Society as a Complex System, una subvención estadounidense para David Lane, Sende van der Leeuw y Geoffrey West, con un subcontrato para el autor. ISCOM financió igualmente la asistencia para la investigación de Joseph Wehbe. La primera presentación de las ideas de la teoría de la cohesión circular en el verano de 2003 se benefició del apoyo del Santa Fe Institute para el encuentro anual de ISCOM. Andrej Mrvar respondió amablemente a mi petición subsiguiente de ampliar la capacidad de búsqueda fragmental de Pajek automatizando los procedimientos de la cohesión circular, extendiendo los macros, los comandos y las opciones disponibles, y finalmente programando una función de repetición macro. La segunda presentación de la teoría de cohesión circular, específicamente en el contexto del análisis de parentesco con Pajek, fue posible gracias a una invitación de los historiadores de la 
Sorbona, espcialmente de Cyril Grange y sus colegas, para ofrecer un taller de Pajek durante tres días en París en junio de 2004 sobre el análisis de redes de parentesco. Ese taller fue llevado conjuntamente por miembros del grupo de investigación TIME (Traitement Informatique des Matériaux Ethnographiques) para el análisis antropologico del parentesco, encabezado por Laurent Barry y Michael Houseman. Los encuentros sucesivos de aquel grupo en París proporcionaron los avances finales que se requerían para que este método fuera aplicado al parentesco, y condujeran al documento de Hamberger et al. 


\section{BIBLIOGRAFÍA}

Batagel, V., MrVar, A. (1998): «Pajek-A program for large networks analysis», Connections 21(2), Uníversity of Ljubljana, pp. 47-57. http://vlado.fmf.unilj.si/pub/networks/pajek. Para una descripción del programa, ver: http://vlado.fmf.unilj.si/pub/networks/pajek/pajek. pdf

- (2001): «A subquadratic triad census algorithm for large sparse networks with small maximum degree», Social Networks 23, pp. 237-243.

- (2002): «Pajek-Analysis and visualization of large networks», LNCS 2265: Graph Drawing, 9th International Symposium, Berlin, Springer, pp. 477-478.

- (2003): «Pajek analysis and visualization of large networks», Graph Drawing Software, M. Junger and P. Mutzel (eds.), Berlin, Springer (series in Mathematics and Visualization), pp. 77-103. http://www.ijp.si/ftp/pub/preprints/ps/2003/ pp871.pdf

- (2004): «Relinking marriages in genealogies», Metodolo ki zvezki - Advances in Methodology and Statistics 1, Ljubljana, FDV, pp. 407-418.

BATAGEL, V., ZAVERSNIK, M. (2003): «Short cycles connectivity», CoRR cs.DS/0308011, pp. 1-21.

BRUDNER, L. A, WhITE, D. R. (1997): «Class, property and structural endogamy: Visualizing networked histories», Theory and Society 25, pp. 161-208.

BERTIN, J. (1983): Semiology of Graphics, Trans. William J Berg; Madison (WI), University of Wisconsin Press.

BuRT, R. S. (1995): Structural Holes: The Social Structure of Competition, Boston, Harvard University Press.

Das, T. C. (1945): The Purums, Calcutta, University of Calcutta Press.

De NoOY, W., MRVAR, A., BATAGEL, V. (2004): Exploratory Social Network Analysis with Pajek, New York, Cambridge University Press.

GoldENWEISER, A. A. (1913): «The origins of totemism», American Anthropologist N.S. 14, pp. 530-562.

Hamberger, K., Houseman, M., Datllent, L., White, D. R., BarRy, R. (2004): «Matrimonial ring structures», Mathématiques, Informatique, et Sciences Humaines 168.

HARARY, F. (196): Graph Theory. Reading (MA), Addison-Wesley.

Hèran, F. (1995): Figures et légendes de la parenté, París, INED.

HARARY, F., WhITE, D. R. (2001): «The Cohesiveness of Blocks in Social Networks: Node Connectivityand Conditional Density», Sociological Methodology 2001 31(1), Boston, Blackwell Publishers, pp. 305-359.

HousemAN, M., WHTTE, D. R. (1998a): «Network mediation of exchange structures: Ambilateral sidedness and property flows in Pul Eliya», Kinship, Networks and Exchange, T. Schweizer and D. R. White (eds.), Cambridge, Cambridge University Press, pp. 59-89.

- (1998b) «Taking sides: Marriage networks and Dravidian kinship in lowland South America», Transformations of Kinship, M. Godelier, T. Trautmann, and F. Tjon Sie Fat (eds,), Washington (D.C.), Smithsonian Institution Press, pp. 214-243.

JOHANSEN, U. C., WhITE, D. R. (2002): «Collaborative long-term ethnography study and longitudinal social analysis of a nomadic clan in Southeastern Turkey», Chronicling Cultures: Long-Term Field Research in Anthropology, R. Kemper and A. Peterson Royce (eds), Walnut Creek (CA), AltaMira Press, pp. 81-99.

Jola, T., VerdiER, Y., ZONABEND, F. (1970) «Parler famille», L'Homme 10(3), pp. 5-26. 
JORION, P. (1984): «L'inscription dans la structure de parenté: Omicar?», Revue du Champ Freudien 31, pp. 56-97.

Levi-STRAuss, C. (1958): Anthropologie structurale, Paris, Plan.

- (1963): «Linguistics and anthropology», Structural Anthropology, New York, Basic Books, pp. 67-97.

- (1969): The Elementary Structures of Kinship, London, Eyre and Spottiswoode. [Trans. of Les Structures Elémentaires de la Parenté, París, PUF, 1949].

Menger, K, (1927): «Zur Allgemeinen Kurventheorie», Fundamenta Mathematicae 10 , pp. 96-115.

Moody, J., WHITE, D. R. (2003): «Social cohesion and embeddedness: A hierarchical conception ofsocial groups", American Sociological Review 68, pp. 1-25. http://www.asanet.org/journals/ASRFeb03MoodyWhite.pdf

ORE, O. (1960): «Sex in graphs», Proceedings of the American Mathematical Society 11, pp. 533-539.

SCHNEIDER, D. (1965): «Some muddles in the models: Or how the system really works», The Relevance of Models for Social Anthropology, M. Banton (ed.), ASA Monograph n..$^{\circ}$, London, Tavistock, pp. 25-85.

VISMARA P. «Union of all the minimum cycle bases of a graph», Electronic Journal of Combinatorics 4, 1997, p. 73-87.

WEL, A. (1969): «On the algebraic study of certain types of marriage laws», Appendix to Part One, C. Lévi-Strauss, Elementary Structures of Kinship. [Trans. of 1949].

WHITE, D. R. (1997): «Structural endogamy and the network graphe de parenté», Mathématiques, Informatique et Sciences Humaines 137, pp. 101-125. http://eclectic.ss.uci.edu/nsh/str-endo.htm

- (1999): «Controlled simulation of marriage systems», Joumal of Artificial Societies and Social Simulation 3(2). http://jasss.soc.surrey.ac.uk/2/3/5.html

- (n.d.), «The logic of avoidance», Social Networks, [accepted for publication and undergoing author revision].

WhITE, D. R., BATAGELJ, V., MRVAR, A. (1999): «Analyzing large kinship and marriage networks with Pgraph and Pajek», Social Science Computer Review 17, pp. 245-274.

WHITE, D. R., HARARY, F. (2001): «The cohesiveness of blocks in social networks: connectivity and conditional density", Sociological Methodology 2001, Vol. 31, Boston, Blackwell Publishers, pp. 305-359.

WhITE, D. R., HousEMAN, M. (2002): «The navigability of strong ties: small worlds, tic strength and network topology», Complexity 8(1), pp. 72-81. http://repositories.cdlib.orglhcs/DRW2002A

WHITE, D. R., HOUSEMAN, M., SCHWEIZER, T. (1993): «Kinship, social biography and social change database», http://eclectic.ss.uci.edu/ drwhite/PDATASET.htm [serially updated].

White, D. R, Johansen, U. (2004): Network Analysis and Ethnographic Problems: Process Models of a Turkish Nomad Clan, Langham (MD), Lexington Press.

WhITE, D. R, JORION, P. (1992):, «Representing and analyzing kinship: A network approach», Current Anthropology 33, pp. 454-462.

- (1996): «Kinship networks and discrete structure theory: Applications and implications», Social Networks 18, pp. 267-314. 


\section{RESUMEN}

La cohesión circular, como teoría relevante de la cohesión social, se presenta en el análisis de los reencadenamientos matrimoniales como el resultado de una aproximación estructural: «Los estudios estructurales son, en las ciencias sociales, el resultado indirecto de los modernos desarrollos matemáticos que han ido dando cada vez más importancia al punto de vista cualitativo en contraste con el punto de vista cuantitativo de las matemáticas tradicionales. Esto ha hecho posible en campos como la lógica matemática, la teoría de conjuntos, la teoría de grupos y la topología, el desarrollo de una aproximación rigurosa a los problemas que no admiten una solución métrica. Los destacados logros en esta conexión -que ofrecen por sí mismos un trampolín todavía no utilizado por el científico social-se encuentran en Theory of Games and Economic Behaviour de J. von Neumann y $O$. Morgenstern, Cybernetics de $N$. Wiener $y$ The Mathematical Theory of Communication de C. Shannon y W. Weaver». [LéviStrauss, Structural Anthropology, 1963, Capítulo XV, Social Structure, sección de «Structure and Measure», p. 283].

\section{PALABRAS ClaVe}

Red de parentesco, Reencadenamientos de alianzas, Cohesión social, Endogamia estructural.

\section{ABSTRACT}

Ring cohesion, as a theory relevant to social cohesion, offers itself in the analysis of matrimonial relinking as an outgrowth of a structural approach: "Structural studies are, in the social sciences, the indirect outcome of modern developments in mathematics which have given increasing importante to the qualitative point of view in contradistinction to the quantitative point of view of tradicional mathematics. It has become possible, therefore, in fields such as mathematical logic, set theory, group theory, and topology, to develop a rigorous approach to problems which do not admit of a metrical solution. The outstanding achievements in this connection -which offer themselves as springboards not yet utilized by social scientist-is to be found in J. von Neumann y O. Morgenstern, Theory of Games and Economic Behaviour; $N$. Wiener, Cybernetics; and $C$. Shannon and $W$. Weaver, The Mathematical Theory of Communication». [Lévi-Strauss, Structural Anthropology, 1963, Capítulo XV, Social Structure, sección de «Structure and Measure», p. 283].

\section{KEY WORDS}

Kinship network, Family relinking, Social cohesion, Structural endogamy. 